\title{
Asymptotic Models for Tropical Intraseasonal Oscillations and Geostrophic Balance ${ }^{\mathscr{A}}$
}

\author{
SAMUEL N. STECHMANN \\ Department of Mathematics, and Department of Atmospheric and Oceanic Sciences, University of Wisconsin-Madison, \\ Madison, Wisconsin \\ SCOTT HOTTOVY \\ Department of Mathematics, United States Naval Academy, Annapolis, Maryland
}

(Manuscript received 6 June 2019, in final form 9 March 2020)

\begin{abstract}
In the tropics, rainfall is coupled with waves in the form of, for example, convectively coupled equatorial waves (CCEWs) and the Madden-Julian oscillation (MJO). In perhaps the simplest viewpoint of CCEWs, the effects of moisture and convective adjustment can predict the basic aspects of their propagation and structure: reduced propagation speeds and reduced meridional length scales. Here, a similar simple viewpoint is investigated for the MJO's propagation and structure. To do this investigation, budget analyses of a model MJO are first presented to illustrate and motivate the asymptotic scaling assumptions. Asymptotic models are then derived for the MJO. In brief, the structure of the asymptotic MJO is described by a tropical geostrophic balance, and the slow propagation arises from the dynamics of moist static energy. To be specific, if the moist static energy has a background vertical gradient that is asymptotically weak (i.e., a moist stability that is nearly neutral), then it supports a slowly propagating wave. Beyond these main aspects, other processes also have an influence, such as eddy diffusion of moisture. In comparing the simple viewpoints of CCEWs and the MJO, one main difference is in the propagation speeds: relative to a dry wave speed of $50 \mathrm{~m} \mathrm{~s}^{-1}$, the MJO has a speed of $5 \mathrm{~m} \mathrm{~s}^{-1}$, resulting from a reduction factor of 0.1 related to moist stability, whereas the basic CCEW speed is $15 \mathrm{~m} \mathrm{~s}^{-1}$, resulting from a reduction factor of the square root of 0.1 , related to the square root of the moist stability.
\end{abstract}

\section{Introduction}

The Madden-Julian oscillation (MJO) is the dominant mode of tropical intraseasonal variability (Madden and Julian 1971, 1972, 1994; Zhang 2005), and it has significant impacts on global climate. For example, it interacts with tropical cyclones (Liebmann et al. 1994; Maloney and Hartmann 2000), El Niño-Southern Oscillation (Hendon et al. 2007), active and break phases of monsoons (Lau and Waliser 2012), midlatitude weather and its predictability (Jones 2000; Jones et al. 2004), and ocean biogeochemistry (Waliser et al. 2005).

This 30-90-day cycle is one of the last major atmospheric phenomena that is not well understood (e.g., Randall 2012;

\footnotetext{
Supplemental information related to this paper is available at the Journals Online website: https://doi.org/10.1175/JCLI-D-190420.s1.
}

Corresponding author: Scott Hottovy, hottovy@usna.edu
Zhang et al. 2013). To contribute to further understanding of the dynamics and physics of the MJO, the main question of study in the present paper is, Can an asymptotic model be derived to embody some of the basic mechanisms of the MJO and/or tropical intraseasonal variability?

Our motivation for studying this question is from comparison with the case of convectively coupled equatorial waves (CCEWs). In the case of CCEWs, as a simple model, one could consider the dynamics of velocity $\mathbf{u}=$ $(u, v)$, potential temperature $\theta$, and moisture $q$ as

$$
\begin{aligned}
\frac{\partial \mathbf{u}}{\partial t}+y \mathbf{u}^{\perp}-\nabla \theta & =0, \\
\frac{\partial \theta}{\partial t}-\nabla \cdot \mathbf{u} & =\frac{1}{\tau} q, \quad \text { and } \\
\frac{\partial q}{\partial t}+\tilde{Q} \nabla \cdot \mathbf{u} & =-\frac{1}{\tau} q,
\end{aligned}
$$

where $\tau$ is the time scale of convective adjustment (e.g., Gill 1982b; Neelin and Yu 1994; Frierson et al. 2004; 
Ogrosky and Stechmann 2016). In the limit of fast convective adjustment or strict quasi equilibrium, one finds that convergence and precipitation are in balance (i.e., $\tilde{Q} \nabla \cdot \mathbf{u}=-q / \tau)$, and the asymptotic dynamics become

$$
\begin{aligned}
\frac{\partial \mathbf{u}}{\partial t}+y \mathbf{u}^{\perp}-\nabla \theta & =0 \quad \text { and } \\
\frac{\partial \theta}{\partial t}-(1-\tilde{Q}) \nabla \cdot \mathbf{u} & =0 .
\end{aligned}
$$

This asymptotic model embodies two simple predictions: the structure of CCEWs, which propagate zonally, should have a reduced meridional length scale, reduced by a factor of $(1-\tilde{Q})^{1 / 2}$, and CCEWs should have a reduced phase speed $c$ :

$$
c_{\mathrm{CCEW}}=\sqrt{1-\tilde{Q}} c_{\mathrm{dry}} .
$$

While this asymptotic model is not perfect in every way (e.g., Kiladis et al. 2009), it provides a simple viewpoint of CCEWs.

In this paper, a main goal is to explore a similar simple viewpoint for the MJO. In the simple theory of CCEWs described above, the main components of the model are the vertical gradient of background moisture $\tilde{Q}$ and the convective adjustment time $\tau$. A similar perspective, but focused on this paper's topic of the MJO, could potentially come from the model of Stechmann and Hottovy (2017). It involves a similar backbone, and it was shown to be able to represent CCEWs, the MJO, and the background spectrum of tropical convection. Therefore, it will be used in this paper as a starting point in the analysis of an asymptotic model for the MJO, in much the same way that (1) provided the starting point for an asymptotic model for CCEWs.

Just as (1)-(3) is not a perfect model of CCEWs, a similar viewpoint of the MJO will also not be perfect in every way. Beyond the processes considered herein, many additional processes have also been examined in the past in relation to the MJO, and they could potentially be included in the future as additional processes. For example, additional contributions could come from boundary layer frictional convergence (Wang and Rui 1990; Salby et al. 1994; Wang et al. 2016), evaporationwind feedback or wind-induced surface heat exchange (WISHE) (Emanuel 1987; Neelin et al. 1987), cloudradiative feedbacks, moisture-mode theories (Raymond and Fuchs 2009; Sobel and Maloney 2013; Adames and Kim 2016), water vapor-convective activity feedback as in the skeleton model (Majda and Stechmann 2009b), or multicloud models including stratiform and congestus clouds (Khouider et al. 2011; Deng et al. 2015). In the present paper, many additional processes will be discussed in section 5, although the main goal is to use a small number of processes, similar to the simple CCEW model described in (1), but here for the MJO. Models of such limited complexity will naturally not be perfect representations of nature, as, for example, the CCEW model in (1) misses the vertical tilts of real CCEWs (e.g., Wheeler and Kiladis 1999; Mapes 2000; Khouider and Majda 2006; Kuang 2008; Kiladis et al. 2009). Nevertheless, models of limited complexity can be valuable for encapsulating simple processes and, for example, explaining the reduced phase speeds of moisture-coupled waves.

In addition, beyond the simplest types of models, more complex models are also considered. These results are shown in section 5. There, several examples are given in more general setups where many additional processes can be included and nonlinear terms are considered.

The outline of the paper is as follows: Section 2 describes the first model that we consider, and section 3 describes the associated budget analysis of the model MJO. The budget analysis describes the main mechanisms of the model MJO, and it illustrates and motivates the asymptotic scaling assumptions. In section 4 , an asymptotic analysis is performed to derive simplified models for the MJO. In section 5, several additional models and processes are discussed. Conclusions are summarized in section 6 .

\section{Model description}

In this paper, a hierarchy of different models will be considered, including a variety of different formulations and processes. Many of them will be described in the later sections 4 and 5 . In this section, we present the model that will be used later in budget analyses (in later section 3) and that provided our own motivation for the asymptotic analyses.

The starting point here is the model from Stechmann and Hottovy (2017). The nondimensional model equations are

$$
\begin{gathered}
\frac{\partial \mathbf{u}}{\partial t}+y \mathbf{u}^{\perp}-\nabla \theta=-\frac{1}{\tau_{u}} \mathbf{u} \\
\frac{\partial \theta}{\partial t}-\nabla \cdot \mathbf{u}=\frac{1}{\tau_{\text {low }}} q_{\text {low }}+\frac{1}{\tau_{\text {mid }}} q_{\text {mid }}-\frac{1}{\tau_{\theta}} \theta, \\
\frac{\partial q_{\text {low }}}{\partial t}+\tilde{Q}_{\text {low }} \nabla \cdot \mathbf{u}=-\frac{1}{\tau_{\text {low }}} q_{\text {low }}+b_{\text {low }} \nabla^{2} q_{\text {low }}, \quad \text { and } \\
\frac{\partial q_{\text {mid }}}{\partial t}+\tilde{Q}_{\text {mid }} \nabla \cdot \mathbf{u}=-\frac{1}{\tau_{\text {mid }}} q_{\text {mid }}+b_{\text {mid }} \nabla^{2} q_{\text {mid }},
\end{gathered}
$$

where $\mathbf{u}=(u, v)^{\mathrm{T}}$ is the vector of zonal and meridional velocity components, respectively, of the first vertical 
TABLE 1. Standard parameter values for the model in (4).

\begin{tabular}{lcc}
\hline \hline Parameter & Dimensionless value & Dimensional value \\
\hline$\tau_{\text {low }}$ & $1 / 2$ & $4 \mathrm{~h}$ \\
$\tau_{\text {mid }}$ & 4 & 1.33 days \\
$\tau_{u}=\tau_{\theta}$ & 48 & 16 days \\
$\tilde{Q}_{\text {low }}$ & 0.9 & - \\
$\tilde{Q}_{\text {mid }}$ & 0.45 & - \\
$b_{\text {low }}$ & 0.1 & $7.58 \mathrm{~km}^{2-1}$ \\
$b_{\text {mid }}$ & 0.8 & $60.6 \mathrm{~km}^{2} \mathrm{~s}^{-1}$ \\
\hline
\end{tabular}

baroclinic mode, $\theta$ is the first baroclinic potential temperature, and $q_{\text {low }}$ and $q_{\text {mid }}$ are the mixing ratios of water in the lower and middle troposphere, respectively. The dynamical core on the left-hand side can be derived from the $3 \mathrm{D}$ primitive equations using projections onto vertical basis functions [see the supplemental information of Stechmann and Hottovy (2017)]. The Coriolis term is written, under the equatorial betaplane approximation, as $y \mathbf{u}^{\perp}$, where $\mathbf{u}^{\perp}=(-v, u)^{\mathrm{T}}$. The equations have been nondimensionalized using standard equatorial reference scales following, for example, Stechmann et al. (2008), Majda and Stechmann (2009a), and Stechmann and Majda (2015) that are summarized in Table S1 in the online supplemental material. For the focus of this paper, it is sufficient to neglect both deterministic and stochastic forcing and to consider the unforced version of the model, as shown in (4). The standard (dimensional) values of the parameters for the model are given in Table 1.

A brief description of the model processes is as follows, and further details can be found in Stechmann and Hottovy (2017). The processes of the model include the $\beta$-plane approximation to the Coriolis effect, moisture convergence (through the convergence terms $\tilde{Q}_{\text {low }} \nabla \cdot \mathbf{u}$ and $\left.\tilde{Q}_{\text {mid }} \nabla \cdot \mathbf{u}\right)$, convective adjustment terms $q_{\text {low }} / \tau_{\text {low }}$ and $q_{\text {mid }} / \tau_{\text {mid }}$ at two vertical levels, and Rayleigh momentum damping $-\mathbf{u} / \tau_{u}$ and Newtonian cooling $-\theta / \tau_{\theta}$, which are linear (e.g., Kuang 2012) with long time scales (16 days) that are consistent with results in Stechmann and Ogrosky (2014). Note that this is a linear model, but it does include a representation of nonlinear moisture advection by eddies, in the form of eddy diffusion, $\nabla^{2} q_{\text {low }}$ and $\nabla^{2} q_{\text {mid. }}$.

With regard to horizontal moisture advection, for the MJO, one could consider two contributions: planetaryscale advection versus eddy advection. The model in (4) is linear, and it includes the effects of eddy advection but not nonlinear planetary-scale advection. The use of a linear model will simplify the analysis and is a common simplifying assumption in past work as well (e.g., Neelin and Yu 1994; Khouider and Majda 2006). Nonlinear advection could be explicitly included in a more complex version of the model, and it is discussed below in section 5 .
In any case, it is eddy advection that is arguably more important (e.g., Maloney 2009; Kiranmayi and Maloney 2011; Andersen and Kuang 2012). In a simple model, Sobel and Maloney (2013) represent the meridional transport of moisture, due to synoptic-scale eddies, as $-\partial_{y} \overline{v^{\prime} q^{\prime}}=-D u$, where $D$ is a constant-that is, it is assumed to be proportional to MJO zonal wind anomaly $u$ (at the equator $y=0$ since their model is a onedimensional model above the equator and does not include variations in $y$ ). In contrast, here meridional advection of moisture is modeled as $-\partial_{y} \overline{v^{\prime} q^{\prime}}=b \partial_{y}^{2} q$, as eddy diffusion.

The two vertical levels of moisture, $q_{\text {low }}$ and $q_{\text {mid }}$, can also be related to two vertical modes. The use of one vertical mode is somewhat common for moisture (e.g., Neelin and Yu 1994; Khouider and Majda 2006; Sobel and Maloney 2013), and the addition of a second vertical mode here allows further details of the vertical structure seen in observations (Kiladis et al. 2005, 2009). The relationship between the vertical levels and vertical modes is similar to a discrete Fourier transform (see Stechmann and Hottovy 2017, their supplemental information). In terms of vertical levels, $q_{\text {low }}$ and $q_{\text {mid }}$ are coupled through the circulation: $q_{\text {low }}$ and $q_{\text {mid }}$ act as heat sources in the convective adjustment terms, thereby driving circulations that feed back on $q_{\text {low }}$ and $q_{\text {mid }}$ evolution via the convergence terms $\tilde{Q}_{\text {low/mid }} \nabla \cdot \mathbf{u}$. Also note that two convective adjustment times are used, $\tau_{\text {low }}$ and $\tau_{\text {mid }}$, for the lower and middle troposphere, respectively, and $\tau_{\text {mid }}$ is a slower time scale of roughly 1-4 days. The use of two different adjustment times, with slower response in the mid-upper troposphere, was needed to produce a unified spectrum of tropical variability (including CCEWs, MJO, and background spectrum together) in past versions of this model (Stechmann and Hottovy 2017; Ogrosky et al. 2019). This slower response is in agreement with other studies of convective adjustment (Raymond and Herman 2011), and it is also consistent with the time scale of roughly 1 to 4 days that can be identified with the background spectrum of tropical convection (Takayabu 1994; Wheeler and Kiladis 1999; Hottovy and Stechmann 2015).

The model in (4) has numerous eigenmodes, and one of the eigenmodes - the least damped eigenmoderesembles the MJO. In Fig. 1 the horizontal (Fig. 1a) and vertical structure (Fig. 1b) of the mode are plotted. The eigenmode has zonal convergence of winds near the maxima of rainfall on the equator, as well as off-equatorial quadrupole gyres. The vertical structure shows the overturning circulation. In Fig. 1c, the dispersion relationship is shown on top of the anomalous power spectrum (Stechmann and Hottovy 2017). The MJO eigenmode is 

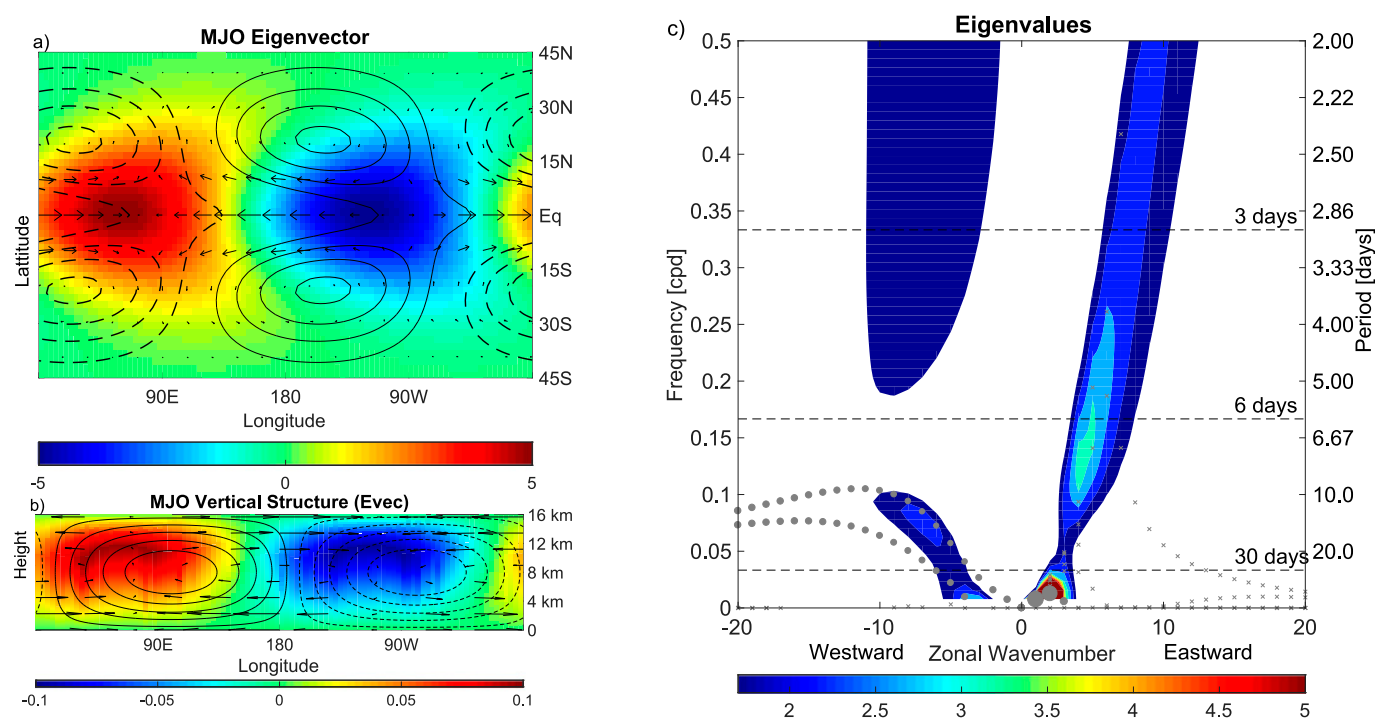

FIG. 1. The MJO eigenvectors of the model in (4) and the dispersion relation: (a) The MJO eigenmode horizontal structure, with precipitation $\left(\mathrm{mm} \mathrm{day}^{-1}\right.$; colors), lower-tropospheric wind vectors, and lower-tropospheric geopotential height anomalies for the eigenvector (contours; interval is $4.33 \mathrm{~m}$, with positive contours being solid black and negative contours being dashed). (b) The MJO eigenmode vertical structure, with water vapor mixing ratio ( $\mathrm{g} \mathrm{kg}^{-1}$, colors), zonal-vertical velocity vectors, and potential temperature anomalies for the eigenvector (contours; interval is 0.04). (c) The dispersion relation for all modes of the model, with the size of the dots being proportional to the inverse of the growth rate. The MJO modes are the largest circles for $k=1,2$, and 3 for frequencies less than 30 days.

the series of larger dots on top of the spectral power peak at wavenumbers $k=1,2$, and 3 .

The standard parameter regime in Stechmann and Hottovy (2017) was chosen to give the best unified case of the MJO and CCEW. For this regime, the decay scale of the MJO is 48 days, and the linear phase speed is $3.5 \mathrm{~m} \mathrm{~s}^{-1}$, whereas the nonlinear phase speed is $4 \mathrm{~m} \mathrm{~s}^{-1}$ (Ogrosky et al. 2019). Changing the various parameters of the model will cause various changes to the eigenmodes of the system. Different parameter regimes are shown in Stechmann and Hottovy (2017) and Ogrosky et al. (2019). For example, the speed could be faster or slower, or the MJO could appear at different length scales. One other model that also produces a large-scale $\mathrm{MJO}$ and equatorial waves is the model of Yang and Ingersoll $(2013,2014)$, which also includes clear spectral signals along gravity wave dispersion curves. One similarity is that both models involve some triggering or forcing from small scales, with some differences in formulation: triggering from a deterministic geopotential height threshold in Yang and Ingersoll $(2013,2014)$ and a stochastic moist forcing in Stechmann and Hottovy (2017). Another difference is that Yang and Ingersoll $(2013,2014)$ use a dry system without moisture, whereas Stechmann and Hottovy (2017) use a moist system.

Why were two levels of moisture needed by Stechmann and Hottovy (2017), rather than a single moisture level or a column-integrated moisture? Several physical features can be represented in finer detail if two moisture variables are used, such as a vertical moisture gradient at two levels $\left(\tilde{Q}_{\text {low }}\right.$ and $\left.\tilde{Q}_{\text {mid }}\right)$, different convective adjustment times $\left(\tau_{\text {low }}\right.$ and $\left.\tau_{\text {mid }}\right)$, and different moisture anomalies at different levels $\left(q_{\text {low }}\right.$ and $\left.q_{\text {mid }}\right)$. Moisture vertical structure is sometimes identified as an important element of the MJO in nature and in climate model simulations (e.g., Klingaman et al. 2015), and two vertical moisture gradient parameters $\left(\tilde{Q}_{\text {low }}\right.$ and $\left.\tilde{Q}_{\text {mid }}\right)$ allow finer details of the vertical structure of moisture (e.g., Holloway and Neelin 2009) in comparison to a single gross moist stability parameter $\tilde{Q}$ (e.g., Neelin and Held 1987; Frierson et al. 2004). These finer details of vertical structure are among the key elements that allow the model to represent both CCEWs and the MJO together. Along these lines, the use of different convective adjustment times ( $\tau_{\text {low }}$ and $\left.\tau_{\text {mid }}\right)$ also allows for different physical processes to be represented, such as a fast adjustment time of 2-4 $\mathrm{h}$ for deep convection and a slower adjustment time of 1-2 days for stratiform rainfall or organized convection, a viewpoint described further by Ogrosky et al. (2019). The distinction between deep convection and stratiform rainfall had also been seen to be important in earlier studies of CCEWs (e.g., Mapes 2000; Khouider and Majda 2006), although a faster adjustment time of roughly $3 \mathrm{~h}$ had been used for stratiform in those past studies, whereas more recent work 
TABLE 2. Budget study of the MJO eigenmode, giving contributions toward $\partial q_{\text {mid }} / \partial t$ values. The real parts are positive contributions to growth and negative contributions to decay of the MJO mode. The imaginary parts are eastward and westward contributions to propagation of the MJO mode. Std indicates "standard."

\begin{tabular}{lcccrrr}
\hline \multicolumn{1}{c}{ Parameter } & $\partial_{t} q_{\text {mid }}$ & $-\tilde{Q}_{\text {mid }} u_{x}$ & $-\tilde{Q}_{\text {mid }} v_{y}$ & $-q_{\text {mid }} / \tau_{\text {mid }}$ & $b_{\text {mid }}\left(q_{\text {mid }}\right)_{x x}$ & $b_{\text {mid }}\left(q_{\text {mid }}\right)_{y y}$ \\
\hline$\tilde{Q}_{\text {mid }}=0.30$ & $-0.0277+0.0156 i$ & $0.7501+0.0738 i$ & $-0.2283-0.0721 i$ & -0.25 & -0.0437 & $-0.2557+0.0139 i$ \\
Std $\left(\tilde{Q}_{\text {mid }}=0.45\right)$ & $-0.0069+0.0159 i$ & $0.7451+0.1477 i$ & $-0.1873-0.1603 i$ & -0.25 & -0.0437 & $-0.2710+0.0286 i$ \\
$\tilde{Q}_{\text {mid }}=0.55$ & $0.0025+0.0141 i$ & $0.7421+0.1785 i$ & $-0.1683-0.1984 i$ & -0.25 & -0.0437 & $-0.2777+0.0340 i$ \\
\hline
\end{tabular}

suggests that adjustment times that are closer to 1 day are associated with better MJO simulations in climate models (Khouider et al. 2011; Jiang et al. 2016). Also note that the names $q_{\text {low }}$ and $q_{\text {mid }}$ are chosen to reflect the altitudes of moisture variations in nature, in roughly the lower and middle troposphere, although in the model these variables actually represent anomalies in roughly the middle and upper troposphere (at heights of roughly 5 and $10 \mathrm{~km}$ ) because an idealized Boussinesq model setup is used for simplicity. Furthermore, on a related note, the vertical moisture structure of the model MJO (in Fig. 1b) has its largest anomalies in the upper troposphere near a height of roughly $10 \mathrm{~km}$, whereas in nature the largest anomalies are in the lower troposphere near a height of roughly $5 \mathrm{~km}$ (e.g., see Figs. 9 and 10 of Kiladis et al. 2005). This is perhaps partly related to the Boussinesq nature of the idealized model, and the corresponding crude background moisture profile (see Fig. S4 of Stechmann and Hottovy 2017). In an extended version of the model with three-dimensional variations, Ogrosky et al. (2019) find that the model MJO has its largest anomalies in the lower troposphere, in closer agreement with the MJO in nature. This is one indication of how some of the aspects of the model MJO can change depending on different parameter choices and model setup.

\section{Mechanisms of the model MJO from budget analyses}

The overall goal of this paper is to pursue asymptotic models for the MJO that are simplified and in the same spirit as fast convective adjustment models for CCEWs. Before proceeding, however, it is useful to first examine the dominant mechanisms of the model in (4) using a budget analysis. The budget analysis will provide some guidance for the subsequent scale analysis (section 4), and it also provides a baseline for comparison with the asymptotic models.

Budget analyses are a useful tool study potential mechanisms of the MJO. Similar budget studies have been performed with different models or settings (Majda and Shefter 2001; Khouider and Majda 2006; Andersen and Kuang 2012).

Here the budget analysis is performed by taking the eigenmode for the $k=1 \mathrm{MJO}$ mode and decomposing it into the various terms of the model equations for midtropospheric moisture in (4c); the results are shown in Table 2. A similar analysis can be done for the lowtropospheric moisture in (4d); the results are shown in Table 3, and they are similar to Table 2, so the subsequent discussion is focused on midtropospheric moisture.

Each term in the budget makes a contribution to the eigenvalue of the MJO mode. The eigenvalue has both real and imaginary parts, as does each budget term. The real part contributes to the growth or decay of the time scale of the mode, and the imaginary part contributes to the oscillation frequency and the propagation speed of the mode.

Figure 2 shows a graphical presentation of the budget study for the standard parameter regime. Figure $2 \mathrm{a}$ shows the phase relationship between the various terms of the $q_{\text {mid }}$ equation, with the total tendency (scaled) shown in the dashed line. Figure $2 b$ shows the contributions of each term toward the growth (positive values) or damping (negative values) of the MJO mode. Figure $2 \mathrm{c}$ shows the contributions of each term toward the propagation eastward (positive values) or westward (negative values) of the MJO mode. In what follows in this section, further details are discussed for the main contributions to growth, eastward propagation, scale selection, and oscillation frequency (section 3a-d),

TABLE 3. As in Table 2 except that contributions are toward $\partial q_{\text {low }} / \partial t$ values.

\begin{tabular}{|c|c|c|c|c|c|c|}
\hline Parameter & $\partial_{t} q_{\text {low }}$ & $-\tilde{Q}_{\text {low }} u_{x}$ & $-\tilde{Q}_{\text {low }} v_{y}$ & $-q_{\text {low }} / \tau_{\text {low }}$ & $b_{\text {low }}\left(q_{\text {low }}\right)_{x x}$ & $b_{\text {low }}\left(q_{\text {low }}\right)_{y y}$ \\
\hline$\tilde{Q}_{\text {mid }}=0.30$ & $-0.0277+0.0156 i$ & $2.9096+0.2887 i$ & $-0.8856-0.28$ & -2 & -0.0055 & $-0.0462+0.0073$ \\
\hline $\operatorname{Std}\left(\tilde{Q}_{\mathrm{mid}}=0.45\right)$ & $-0.0069+0.0159 i$ & $2.7264+0.6081 i$ & $-0.6746-0.6058 i$ & -2 & -0.0055 & $-0.0532+0.0137$ \\
\hline$\tilde{Q}_{\text {mid }}=0.55$ & $0.0025+0.0141 i$ & $2.6445+0.7316 i$ & $-0.5804-0.7331 i$ & -2 & -0.0055 & $-0.0561+0.0156 i$ \\
\hline
\end{tabular}



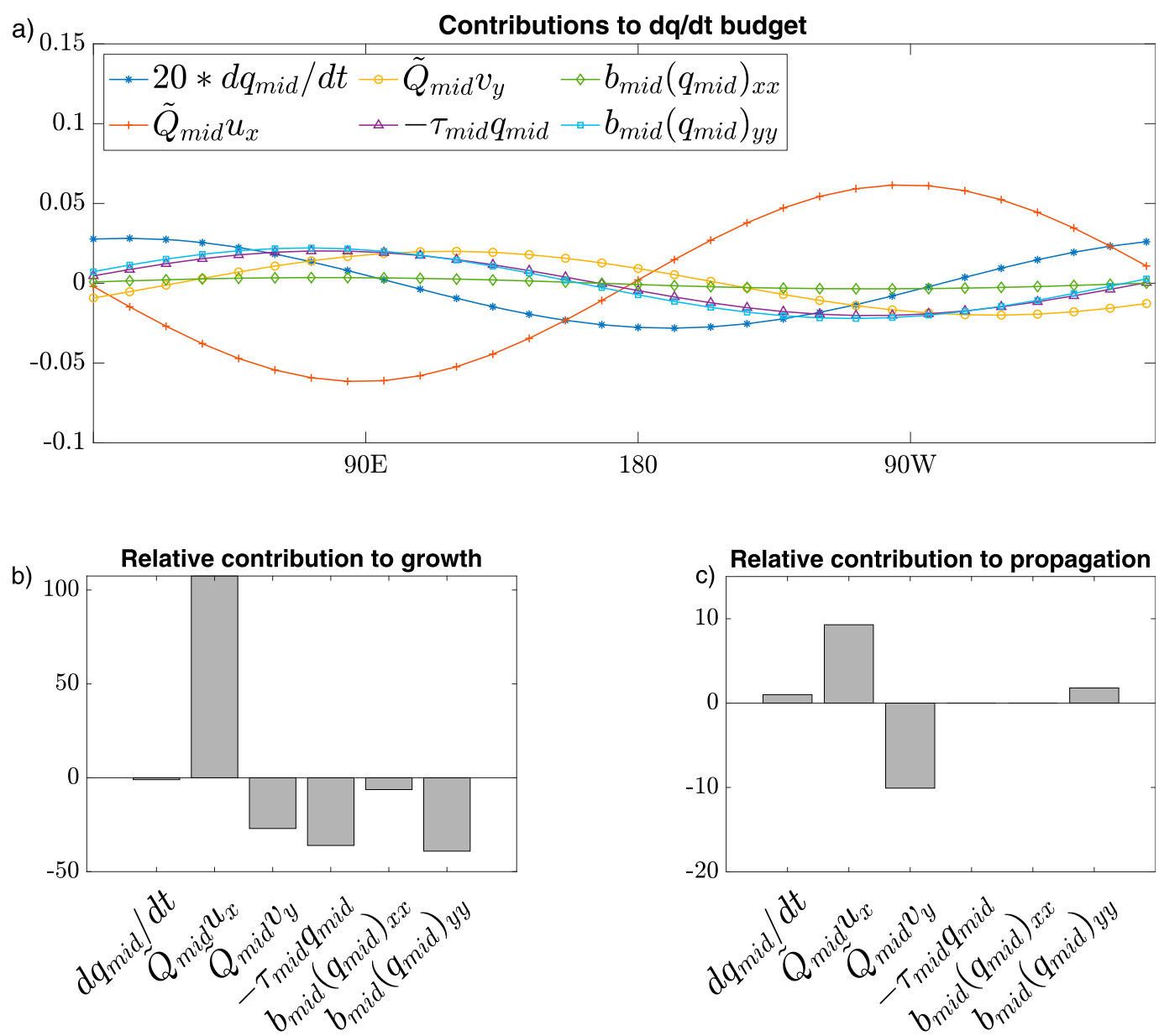

FIG. 2. The terms of the budget analysis: (a) their phase relationships for the $k=1$ wavenumber (the reference is set for the tendency's maximum value at $0^{\circ}$ ) and the numeric contribution to the (b) growth rate and (c) propagation. The nondimensional tendency is -0.0069 for growth and +0.0159 for propagation.

and a summary is described as a growth and propagation sequence (section $3 \mathrm{e}$ ).

\section{a. Growth/decay}

In Fig. 2b, it is shown that the major mechanism for growth is zonal moisture convergence through the term $\tilde{Q}_{\text {mid }} \nabla \cdot \mathbf{u}$. This is balanced by damping through precipitation and meridional divergence of moisture.

Since the largest growth term involves the parameter $\tilde{Q}_{\text {mid }}$, we next ask, How do the processes (e.g., moisture convergence, precipitation) change as $\tilde{Q}_{\text {mid }}$ is varied, and in turn, lead to the changes in the growth rate? Figure 3 shows the budget analysis for the $q_{\text {mid }}$ equation contributions toward growth (Fig. 3a) and propagation (Fig. 3b) of the MJO mode as $\tilde{Q}_{\text {mid }}$ is varied. As $\tilde{Q}_{\text {mid }}$ increases from $\tilde{Q}_{\text {mid }}=0.3$ to $\tilde{Q}_{\text {mid }}=0.55$, the contributions from zonal convergence of moisture, precipitation, and zonal diffusion remain constant. However, meridional divergence of moisture decreases and meridional diffusion increases, and they can impact the growth rate. The stronger of these two effects is from the lessened amounts of meridional divergence, which leads to net growth as $\tilde{Q}_{\text {mid }}$ increases.

For certain parameter choices, the MJO mode even becomes unstable (Stechmann and Hottovy 2017; Ogrosky et al. 2019). For instance, as the environmental moisture gradient $\tilde{Q}_{\text {mid }}$ increases, the MJO can become unstable, as illustrated here for the $\tilde{Q}_{\text {mid }}=0.55$ case in Fig. 3 .

\section{b. Eastward propagation}

Which of the model processes (e.g., moisture convergence, precipitation) are the dominant contributors to the MJO's eastward propagation? In Fig. 2c, the contributions to the propagation of the MJO mode are plotted for each of the middle tropospheric moisture equation terms. The largest term is zonal moisture convergence, which has a maximum slightly to the east of the water vapor maximum (see MJO eigenvector in 
a)

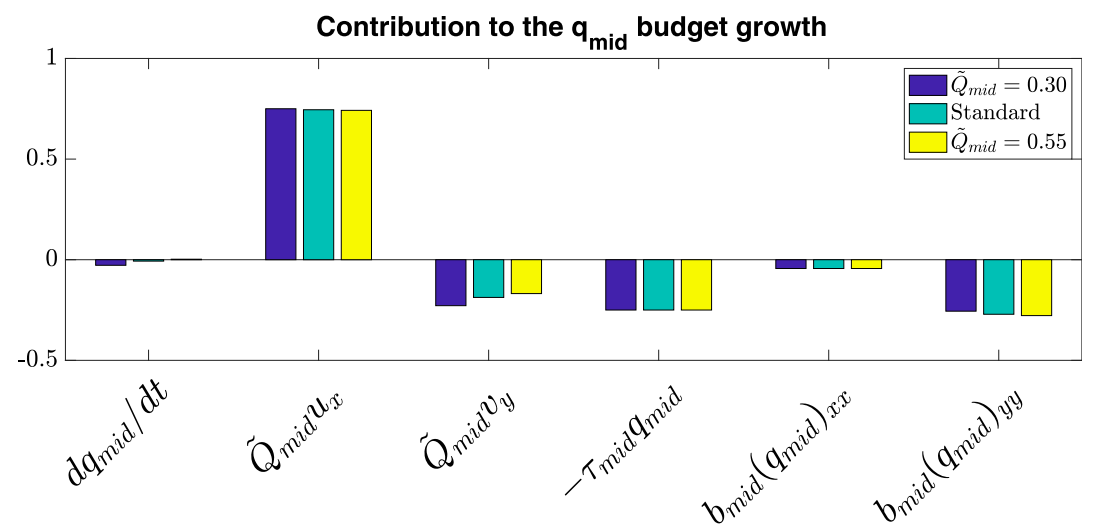

b)

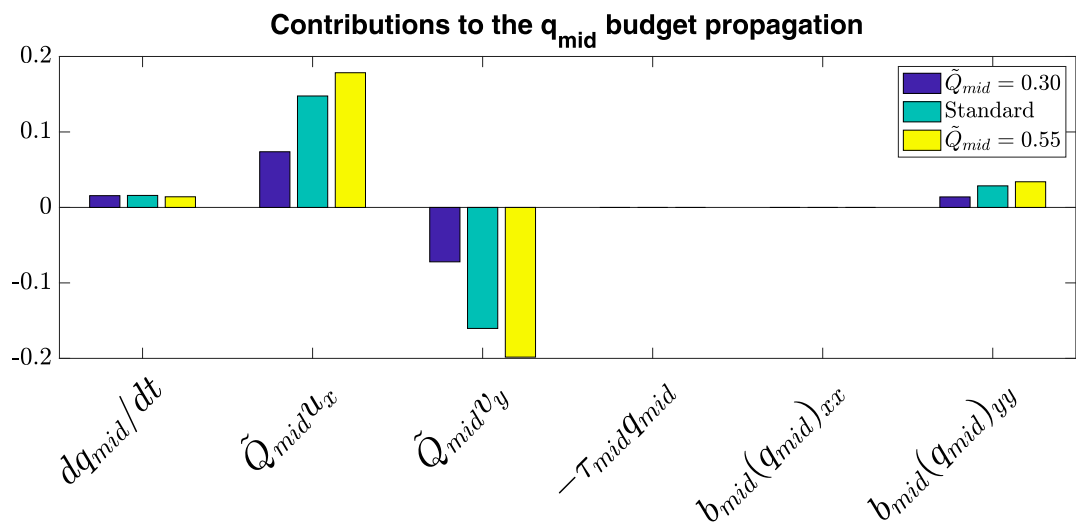

FIG. 3. The terms of the budget analysis are plotted for a variety of values of the strength of convergence of winds $\tilde{Q}_{\text {mid }}$ : the contributions for (a) the growth rate and (b) propagation. The nondimensional values for the tendency for $\tilde{Q}_{\text {mid }}=0.3,0.45$, and 0.55 are $-0.0277,-0.0069$, and +0.0025 , respectively, for the growth and $0.0156,0.0159$, and 0.0141 , respectively, for the propagation.

Fig. 1a) and therefore contributes to eastward propagation. The second largest term is meridional moisture convergence, which has the opposite sign and therefore acts against eastward propagation. A third term is much smaller in magnitude but is able to tip the scales in favor of eastward propagation: meridional eddy diffusion. The terms of precipitation and zonal diffusion have no effect on the propagation.

To explore parameter sensitivity, the effect of varying $\tilde{Q}_{\text {mid }}$ is shown in Fig. 3b. In each case, the zonal and meridional convergence of moisture terms are roughly equal and opposite and would cause a net tendency for slightly westward propagation or no propagation; however, meridional diffusion of moisture makes a slight contribution and brings about slow eastward propagation in each case. Similar results are shown in observational and simulation budget studies of Maloney (2009), Kiranmayi and Maloney (2011), and Andersen and Kuang (2012) that horizontal advection of moisture by meridional eddies plays an important role in the propagation of the MJO.

\section{c. Scale selection}

Why does the MJO appear on planetary length scales? A contrarian question would be, Why is there no MJO at smaller length scales? In terms of the model, this is equivalent to studying the damping rate of the MJO eigenmodes at the different wavenumbers $k$. We explore two possible hypotheses. 1) As the wavenumber increases to smaller scales, the role of diffusion damping $\left(b_{\text {mid }} \partial_{x}^{2} q_{\text {mid }}\right.$, or $b_{\text {mid }} k^{2}$ in Fourier space) becomes much stronger. The other hypothesis is that 2) the Kelvin and Rossby wave structures differ at other length scales in a way that promotes more damping.

To study hypothesis 1 , the effects of diffusion damping $\left(b_{\text {mid }} k^{2}\right)\left(b_{\text {mid }} k^{2}\right)$ are studied in the budget analysis of Fig. 4. Figure 4 shows the contributions to the MJO growth (Fig. 4a) and propagation (Fig. 4b) for the standard parameter regime for different wavenumbers $k$. As $k$ increases, the total tendency for the growth decreases (becomes more damped). This is due to primarily the increase of zonal diffusion $\left[b_{\text {mid }}\left(q_{\text {mid }}\right)_{x x}\right]$. The 
a)

Contributions to the $q_{\text {mid }}$ budget growth

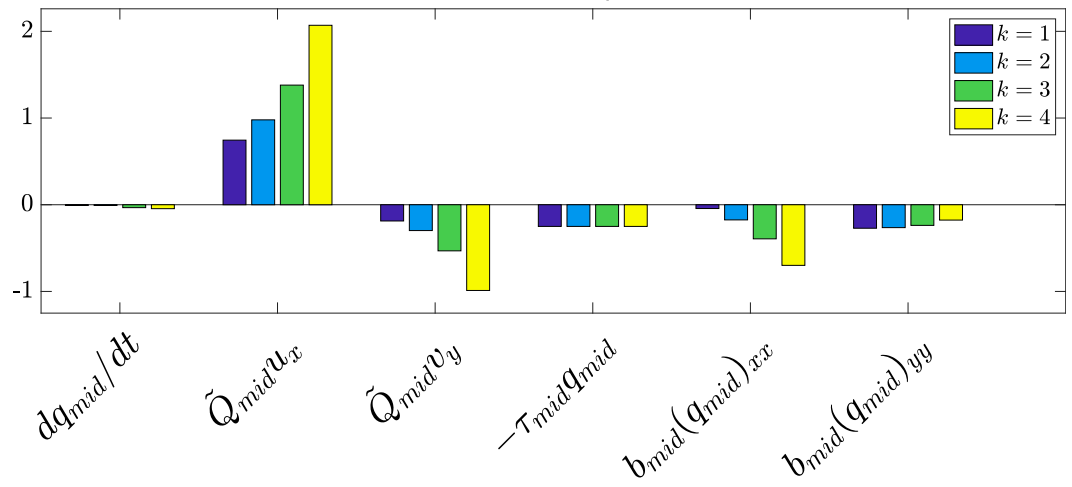

b)

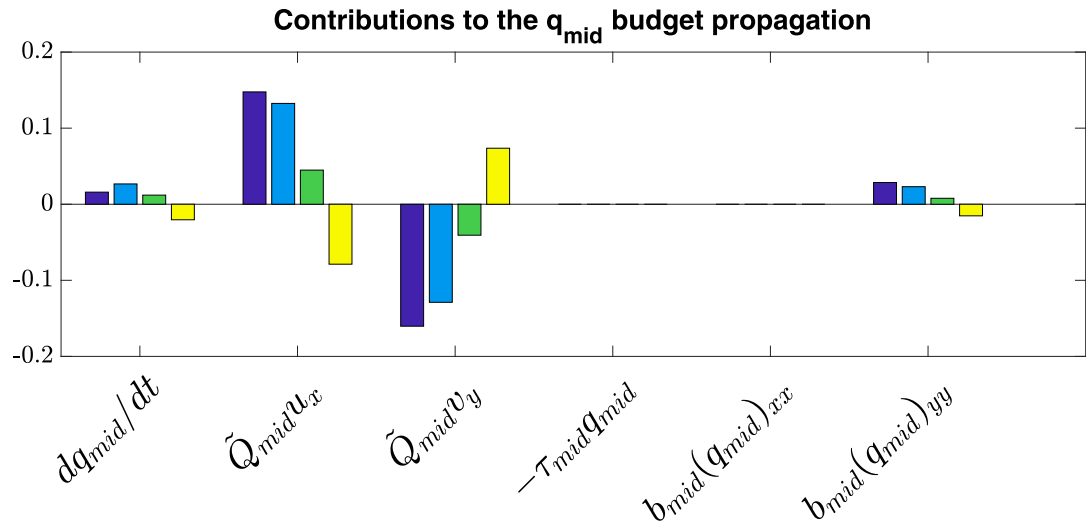

FIG. 4. As in Fig. 3, but showing the budget analysis for wavenumbers $k=1-4$; the nondimensional values for the tendency for wavenumbers $k=1,2,3$, and 4 are $-0.0069,-0.0077$, -0.0335 , and -0.0454 , respectively, for the growth and $0.0159,0.0267,0.0120$, and -0.0204 , respectively, for propagation.

ratio of the contribution of zonal diffusion for $k=1$ versus $k=4$ is much larger than any other term.

To investigate hypothesis 2 , the budget analysis of the MJO eigenmode for different wavenumbers $k$ is considered. Figure 5 shows the horizontal structures for the least damped mode (MJO mode) for wavenumbers $k=1$ (Fig. 5a), $k=2$ (Fig. 5b), $k=3$ (Fig. 5c), and $k=4$ (Fig. 5d). For wavenumbers $k=1$ and $k=2$, the moisture maximum is slightly leading the geopotential height's off-equatorial minima, in terms of their zonal locations $x$ by approximately $1 / 8$ of a cycle. There is also a "nose" to the geopotential height structure near the equator, which is due to competing effects of the Kelvin and Rossby contributions of the MJO's structure. For $k=3$ and $k=4$, the maximum and minimum of moisture are now respectively $90^{\circ}$ out of phase with the maximum and minimum geopotential height. Also, the maxima of moisture have moved in latitude from being equatorial to now being off-equatorial, near the off-equatorial locations of maximum convergence for a dry Rossby wave. Thus, at smaller scales, the MJO eigenmode loses the Kelvin component and the remaining Rossby-like structure is highly damped and westward traveling.

Both factors contribute to the MJO disappearing on smaller length scales. First, the damping rate increases as wavenumber $k$ increases, and therefore the overall variance of this mode is expected to diminish. Second, the eigenmode's propagation actually changes direction; as the wavenumber moves from $k=3$ to $k=4$ (for the standard parameter values), the eigenmode's propagation direction changes from eastward to westward. Concomitantly, the eigenmode's structure changes, with the Kelvin contribution decreasing and the Rossby contribution increasing.

\section{d. Oscillation period}

The oscillation period of the MJO mode is related to several contributing factors. In Fig. 2, bottom panel, the relative contributions of the different middle tropospheric terms are plotted for propagation. Only the convergence of moisture terms $\left[\tilde{Q}_{\text {mid }}\left(u_{x}+u_{y}\right)\right]$ and meridional diffusion contribute to the propagation. From budget analyses alone, the interplay between 

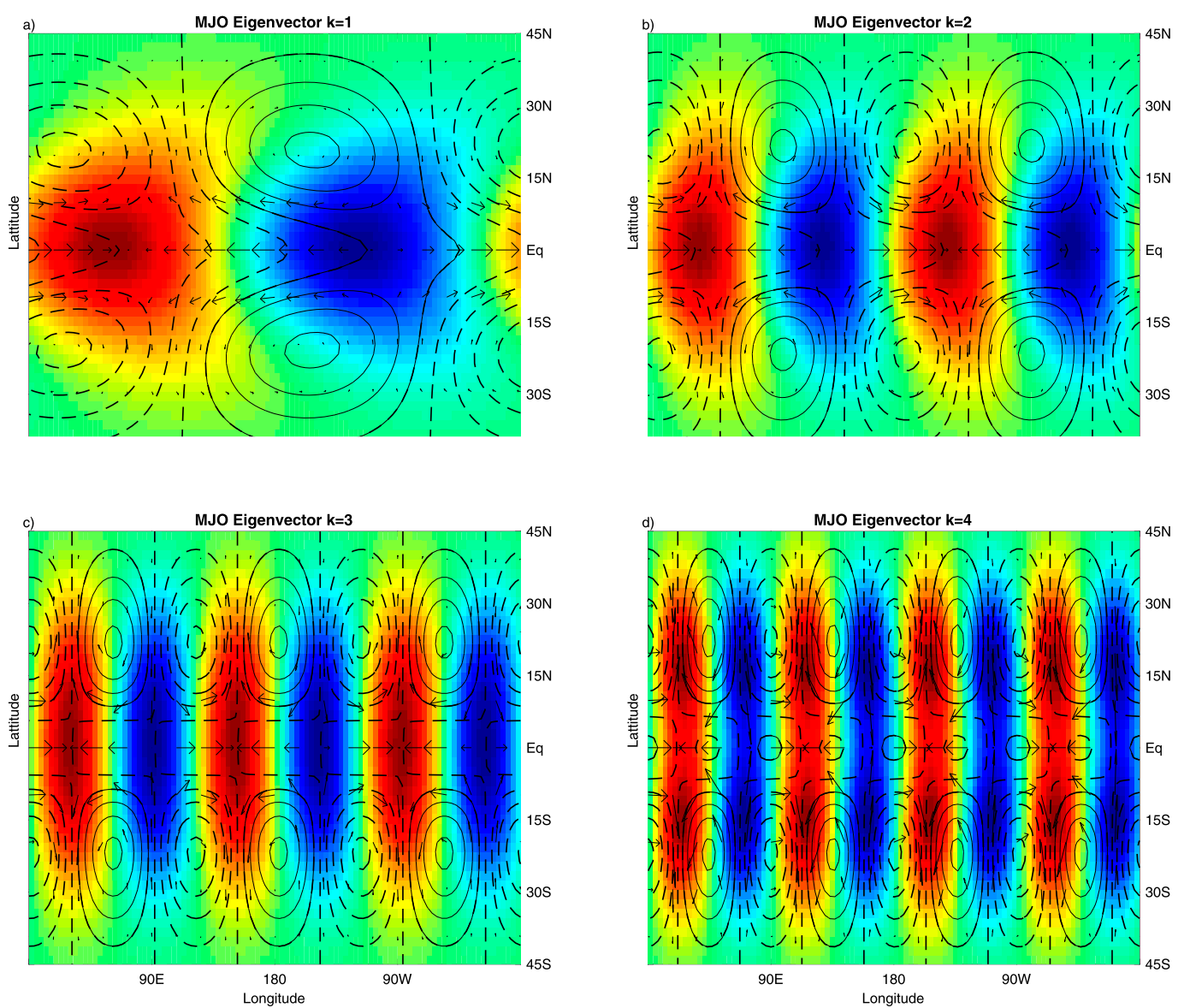

FIG. 5. The horizontal structure for the MJO eigenvector for wavenumbers $k=$ (a) 1 , (b) 2, (c) 3 , and (d) 4 . The shading, vectors, and contours are the same as in Fig. 1.

these parameters to give a realistic oscillation period is not clear. Later in the paper, in section 4, the model is simplified through asymptotic scale analysis, and there it will be shown that the major contributor to the oscillation period is $\left(1-\tilde{Q}_{\text {mid }}-\tilde{Q}_{\text {low }}\right)$.

\section{e. Sequences of growth/decay and propagation}

To summarize the behavior of the model MJO, we illustrate and describe the growth and propagation sequences. To do so, the model is initialized with a large positive anomaly of moisture in the form of a Gaussian function in $x$ and $y$ :

$$
\left(q_{\text {mid }}\right)_{0}=\left(q_{\text {low }}\right)_{0} \propto \exp \left[-(x-\mu)^{2} /\left(2 \sigma^{2}\right)\right],
$$

and zero for all other variables. The model is run for 40 days, and the results are plotted in Fig. 6 in a latitude-longitude plane with moisture (shading), winds (vectors), and geopotential height (contours; negative is dashed) anomalies at $0,1,10$, and 40 days. The evolution illustrates how an initial moisture anomaly excites a propagating MJO.
As a brief overview, from the snapshots in Fig. 6, after one day there is convergence of winds at the center of the moisture anomaly. At 10 days there are noticeable off-equatorial gyres forming to the west of the initial moisture anomaly, associated with a Rossby wave response, and a drying of the atmosphere to the remote east, associated with a Kelvin wave response. From 10 to 40 days, the system has propagated slowly eastward. At day 40 , a pair of anticyclonic gyres has strengthened in the off-equatorial region to the east of the moisture maximum.

The growth can be summarized by the following sequence: a positive anomaly of moisture is associated with convection and a loss of moisture via precipitation; but it simultaneously generates latent heating, which generates a circulation response with significant zonal convergence, a source of moisture. If the zonal convergence of moisture is large enough (i.e., larger than the sum of the moisture loss mechanisms of precipitation, meridional convergence, and eddy diffusion), then the MJO could be unstable. Whether the MJO will be stable 

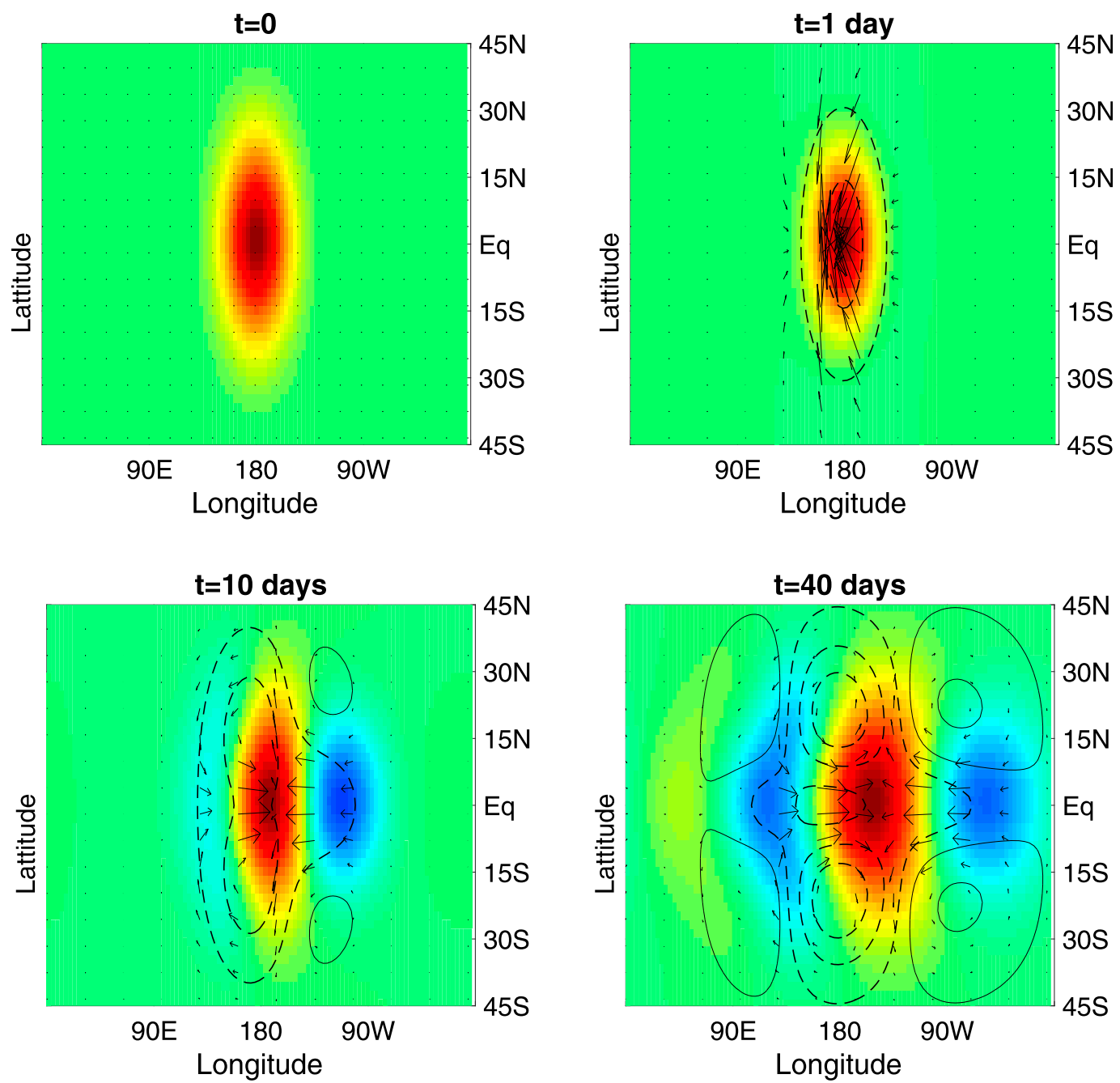

FIG. 6. The initial value problem with a Gaussian initial condition of moisture. The shading, contours, and vectors are the same as in Fig. 1.

or unstable depends on a variety of conditions, such as the strength of the vertical gradient of background water vapor, and the zonal wavenumber (since, e.g., the circulation response to heating depends on zonal wavenumber). Even if the MJO is stable, as in the standard parameter values considered here, it decays quite slowly, and its amplitude is preserved for substantial periods.

The propagation can be summarized by the following sequence. The initial moisture anomaly is associated with precipitation, and simultaneously generates latent heating. This latent heating excites a response in the form of Kelvin and equatorial Rossby waves, in a way that is similar to the gravity wave response to heating (e.g., Bretherton and Smolarkiewicz 1989; Mapes 1993; Stechmann and Majda 2009) except here in the form of equatorial waves. These excited waves are then in competition with each other, with the Kelvin going eastward and the Rossby moving westward, as seen at time $t=$ 10 days in Fig. 6. The circulation patterns of the Kelvin and Rossby waves create moisture convergence that is slightly to the east of the initial moisture anomaly, providing the main contribution to eastward propagation. A smaller effect from meridional eddy diffusion also contributes to eastward propagation. Whether the MJO will propagate eastward or westward depends on a variety of conditions, such as the strength of the vertical gradient of background water vapor, and the zonal wavenumber (since, e.g., the circulation response to heating depends on zonal wavenumber), but eastward propagation prevails for the zonal wavenumber $k=1$ case described here. 


\section{Asymptotic models for the MJO}

The search for asymptotic models is motivated by the budget analyses above, which suggest many balance relations. For example, in Table 3, the time tendency term $\partial_{t} q_{\text {low }}$ is relatively small, and the largest terms in the budget show an approximate balance between moisture convergence, $-\tilde{Q}_{\text {low }}\left(u_{x}+v_{y}\right)$, and precipitation, $-q_{\text {low }} / \tau_{\text {low }}$. Can these balances and others be described by an asymptotic model?

In this section, an asymptotic analysis is conducted for the model in (4). As a prelude, two simplified systems will be studied asymptotically first, including a system with only one moisture variable; the simpler systems help to describe more clearly some of the main components that arise from the full model. One application of the asymptotic models is also presented: they help to elucidate the model mechanisms behind the oscillation period and dispersion relation, which were difficult to analyze in the budget analysis but can be described with more clarity using the asymptotic models.

\section{a. One-vertical-level convective adjustment}

Consider a simpler system with only one vertical level of convective adjustment:

$$
\begin{aligned}
\frac{\partial \mathbf{u}}{\partial t}+y \mathbf{u}^{\perp}-\nabla \theta & =0 \\
\frac{\partial \theta}{\partial t}-\nabla \cdot \mathbf{u} & =\frac{1}{\tau} q, \quad \text { and } \\
\frac{\partial q}{\partial t}+\tilde{Q} \nabla \cdot \mathbf{u} & =-\frac{1}{\tau} q .
\end{aligned}
$$

It will also be useful in what follows to consider an additional equation for moist static energy,

$$
h=\theta+q,
$$

which evolves according to the equation

$$
\frac{\partial h}{\partial t}-(1-\tilde{Q}) \nabla \cdot \mathbf{u}=0 .
$$

Note that dissipation terms have also been neglected here in order to focus more on other aspects such as convective adjustment and moisture convergence. This system has been investigated in the past (e.g., Neelin and $\mathrm{Yu}$ 1994), and a traditional limit of fast convective adjustment, $\tau \rightarrow 0$, leads to a moist wave speed with a reduced value of $1-\tilde{Q}$, in contrast to the dry wave speed of 1 (Gill 1982b; Frierson et al. 2004; Ogrosky and Stechmann 2016).
In contrast to past analyses, what is different about the analysis in this section is the scaling assumptions:

$$
\tau=\varepsilon \quad \text { and } \quad 1-\tilde{Q}=O(\varepsilon),
$$

which also include an assumption that $1-\tilde{Q}$ is small and similar in magnitude to $\tau$. A typical value of $\tilde{Q}$ is 0.9 (e.g., Neelin and Zeng 2000; Khouider and Majda 2006), which leads to $1-\tilde{Q}=0.1$ and suggests that this scaling assumption, while different from past analyses, is reasonably justified.

To proceed with the asymptotic analysis, it is convenient to rescale water vapor and time by defining

$$
q=\varepsilon q^{\prime} \text { and } t^{\prime}=\varepsilon t .
$$

In other words, a long time scale is introduced, and water vapor is expected to be small, since the fast relaxation time scale $\tau=\varepsilon$ will suppress variations in water vapor. The smallness of water vapor may seem unnatural, as it is often not emphasized in presentations of shallow water equations with reduced, moist wave speeds, but the smallness of water vapor does indeed also arise in these contexts for theory of reduced, moist wave speeds as a consequence of fast convective adjustment (e.g., Ogrosky and Stechmann 2016). Note that the result of small of water vapor is removed if additional complexity is introduced into the convective parameterization, such as a nonlinear threshold of $\tau^{-1} \max (0, q)$, and this type of generalization and others are discussed in section 5; here, for the moment, a linearized version of convective adjustment is used to simplify the analysis.

Note that the slow time scale $\varepsilon t$ corresponds to the slow MJO propagation speed of roughly $5 \mathrm{~m} \mathrm{~s}^{-1}$. To see this, start by noting that (6) has been nondimensionalized using the standard synoptic scales of dry equatorial wave theory (e.g., Gill 1982a; Majda 2003). The reference length, time, and velocity are roughly $1500 \mathrm{~km}, 8 \mathrm{~h}$, and $50 \mathrm{~m} \mathrm{~s}^{-1}$, respectively. By rescaling time by a factor of $\varepsilon \approx$ 0.1 , a slower time scale is introduced, along with a corresponding slow velocity of $50 \mathrm{~m} \mathrm{~s}^{-1} \times 0.1=5 \mathrm{~m} \mathrm{~s}^{-1}$, consistent with the MJO. In addition, however, note also that a proper treatment of the MJO should use a longer zonal scale of roughly $15000 \mathrm{~km}$, and actually an even longer time scale of $\varepsilon^{2} t$ corresponding to a 33-day time scale, along with additional rescalings; such a proper treatment is described below in section $4 \mathrm{c}$. For the moment, we use $t^{\prime}=\varepsilon t$ without rescaling $x$, since it allows us to explore the new $1-\tilde{Q}$ scaling in (9) for the MJO, and to compare and contrast with CCEW scaling of $1-\tilde{Q}$, in the simplest setting.

With these scaling assumptions and rescaling of $q$ and $t$, the equations of motion become 


$$
\begin{aligned}
\varepsilon \frac{\partial \mathbf{u}}{\partial t^{\prime}}+y \mathbf{u}^{\perp}-\nabla \theta & =0, \\
\varepsilon \frac{\partial \theta}{\partial t^{\prime}}-\nabla \cdot \mathbf{u} & =q^{\prime}, \quad \text { and } \\
\varepsilon^{2} \frac{\partial q^{\prime}}{\partial t^{\prime}}+\tilde{Q} \nabla \cdot \mathbf{u} & =-q^{\prime} .
\end{aligned}
$$

Notice the $\varepsilon^{2}$ factor in front of $\partial q^{\prime} / \partial t^{\prime}$, which arises from one factor of $\varepsilon$ from each of the rescalings of $q$ and $t$.

From these equations, one can immediately see that the leading-order equations are the balance relations

$$
\begin{aligned}
y \mathbf{u}^{\perp}-\nabla \theta & =0, \\
-\nabla \cdot \mathbf{u} & =q^{\prime}, \quad \text { and } \\
\tilde{Q} \nabla \cdot \mathbf{u} & =-q^{\prime} .
\end{aligned}
$$

These relations include geostrophic balance, related to a small Rossby number, and a balance between convergence and convective heating, related to a small Froude number. The latter balance resembles a weak temperature gradient (WTG) approximation, but it differs from the planetary-scale WTG models of Majda and Klein (2003) since a longer time scale is used here and results in geostrophic balance. The third balance relation is related to an assumption of a relatively strong vertical gradient of background moisture, $\tilde{Q}$, an assumption that is similar to strong stratification or small Froude number but applied to moisture.

Note that the balances in (12b) and (12c) were approximately seen in the budget study of section 3 . For example, in Table 3 the budget for low-level moisture is given, and it shows that the divergence is balanced with low-level precipitation $\left(q_{\text {low }} / \tau_{\text {low }}\right)$.

At first glance, one might wonder whether (12b) and (12c) are inconsistent: How can $q^{\prime}$ be equal to both $-\nabla \cdot \mathbf{u}$ and $-\tilde{Q} \nabla \cdot \mathbf{u}$ at the same time? The two equations are, in fact, consistent, up to $O(\varepsilon)$, since it was assumed that $1-\tilde{Q}=O(\varepsilon)$. Nevertheless, while these two balance relations are consistent, they are also redundant, and the system is therefore not yet closed.

The system can be closed by considering the moist static energy. One can approximate $h=\theta+q \approx \theta$ since $q$ is small, and therefore the moist static energy equation could be written with $h$ replaced with $\theta$ as

$$
\frac{\partial \theta}{\partial t}-(1-\tilde{Q}) \nabla \cdot \mathbf{u}=0,
$$

where no rescaling of time was used. By also including the balance relations

$$
y \mathbf{u}^{\perp}-\nabla \theta=0 \quad \text { and }
$$

$$
\tilde{Q} \nabla \cdot \mathbf{u}=-\frac{1}{\tau} q
$$

where the rescaling of $q$ has now also been removed, a closed system is obtained for the four quantities $\mathbf{u}=$ $(u, v), \theta$, and $q$.

To reveal a propagating wave, it is helpful to rewrite the system to obtain a single partial differential equation (PDE) for $q$ alone. To do this, first note that the curl of the geostrophic balance relations in (13b) leads to

$$
y \nabla \cdot \mathbf{u}=-v,
$$

and the first geostrophic balance relation can itself be written as

$$
\partial_{x} \theta=-y v
$$

Hence one can see that all of the quantities $q, \nabla \cdot \mathbf{u}, v$, and $\partial_{x} \theta$ are proportional to each other, with proportionality factors that depend on $y$. For instance, note that $\partial_{x} \theta$ is related to $q$ as

$$
\partial_{x} \theta=-y^{2} \frac{q}{\tilde{Q} \tau} .
$$

Last, by applying $\partial_{x}$ to (13a), and using in addition the relationship $\tilde{Q} \nabla \cdot \mathbf{u}=-q / \tau$, one finds

$$
\frac{\partial}{\partial t}\left(y^{2} q\right)-(1-\tilde{Q}) \frac{\partial q}{\partial x}=0
$$

which is a single PDE for $q$ alone.

After solving (17) for $q$, all other variables can be found from the balance relations in (13), which are similar to the steady Matsuno-Gill model (Matsuno 1966; Gill 1980) although without damping, as in Stechmann and Ogrosky (2014). In fact, since no damping is present, each variable can be written in terms of $q$ using simple formulas:

$$
\begin{gathered}
\partial_{x} \theta=-y^{2} \frac{q}{\tilde{Q} \tau}, \\
v=y \frac{q}{\tilde{Q} \tau}, \quad \text { and } \\
\partial_{x} u=-2 \frac{q}{\tilde{Q} \tau}-y \frac{\partial_{y} q}{\tilde{Q} \tau} .
\end{gathered}
$$

An illustration of the balance above is shown in Fig. 7. The illustration looks like a mixture of Kelvin and Rossby wave structures, similar to the MJO's structure (e.g., Hendon and Salby 1994).

Note that each of the variables $u, v, \theta$, and $q$ satisfies its own evolution equation of the form in (17), since all of these variables are linearly related to each other through 


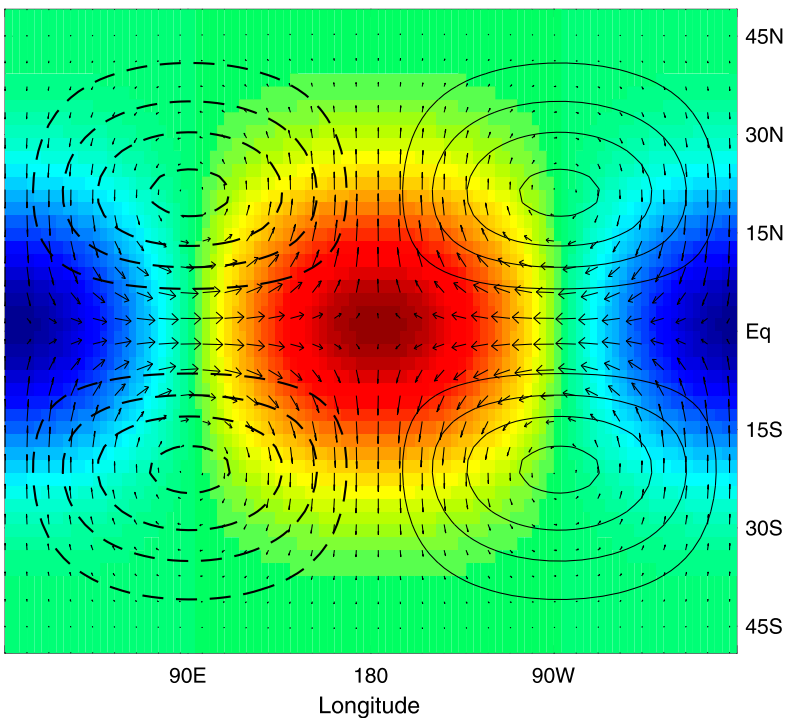

FIG. 7. The MJO eigenvectors resulting from the balance equations in (18). The moisture, $q(x, y)$ was chosen to have the functional form of $q \propto \exp \left(-\alpha_{x} x^{2}-\alpha_{y} y^{2}\right)$, where $\alpha_{x}$ and $\alpha_{y}$ are positive constants. The shading is moisture $q$, the contours are geopotential height $\propto \theta$ (solid lines are positive anomalies, and dashed lines are negative), and vectors are $u$ ( $x$ direction) and $v$ ( $y$ direction) winds.

the balance relations in (18). The formulation of the system in terms of a single evolution equation for $q$ is similar to the approach of some moisture-mode theories (e.g., Sobel and Maloney 2013; Adames and Kim 2016), although other aspects of the models are different here. The choice of using $q$ instead of $u$, $v$, or $\theta$ in (17) is motivated partly by the fact that moisture and convection are often viewed as being central to the MJO, and partly because it is then straightforward to recover all other variables from the undamped Matsuno-Gill model in (13), given the moisture $q$.

A technical note should be mentioned with regard to the balance relations in (13) and the appearance of $y^{2}$ in (17): these equations were derived under the assumption that $y=O(1)$ and variations in $y$ occur on the equatorial synoptic scale of $\approx 1500 \mathrm{~km}$. As a result, it is perhaps best to take a spectral viewpoint of $y$ variations, in terms of parabolic cylinder functions as meridional basis functions (and in terms of different equatorial Rossby waves with different frequencies), rather than viewing $y$ as a coordinate that could take any values such as $\approx 0, \approx 1$, or $\approx \infty$. Such a spectral viewpoint will be taken at the end of section 4, whereas the physical-space viewpoint of $y$ will be used elsewhere for convenience.

Since (17) looks similar to an advection equation, it suggests that the asymptotic model represents a slow wave with propagation velocity

$$
c \sim-(1-\tilde{Q})=O(\varepsilon) .
$$

In dimensional units, using $50 \mathrm{~m} \mathrm{~s}^{-1}$ as a reference velocity and $\varepsilon=0.1$, a slow propagation speed of $O(\varepsilon)$ is of order $5 \mathrm{~m} \mathrm{~s}^{-1}$, similar to the MJO's propagation speed. However, the sign of $-(1-\tilde{Q})$ would be negative, indicating propagation westward, for a typical value of $\tilde{Q}=0.9$, which potentially spoils its relevance for the MJO. Nevertheless, it would potentially correspond to propagation eastward if the gross moist stability, $1-\tilde{Q}$, were negative (e.g., Neelin and Held 1987; Raymond et al. 2009; Inoue and Back 2015b; Klingaman et al. 2015). Other limitations of the one-vertical-level case will be discussed in section $4 \mathrm{~b}$ and $4 \mathrm{c}$ as additional realism is incorporated. In any case, the main point of this first simple case, with only one vertical level of convective adjustment, was to illustrate the asymptotic system that arises from assuming $1-\tilde{Q}=O(\varepsilon)$; in this case, it is seen that a slow mode arises with unidirectional propagation speed related to $1-\tilde{Q}$ itself, $\operatorname{not}(1-\tilde{Q})^{1 / 2}$.

\section{b. Two-vertical-level convective adjustment, with simplified setup}

In the one-level case of section $4 \mathrm{a}$, a slow propagation speed that scales as $-(1-\tilde{Q})$ could be seen, but it potentially is westward. One can move beyond that simple case by using two vertical levels of convective adjustment:

$$
\begin{aligned}
\frac{\partial \mathbf{u}}{\partial t}+y \mathbf{u}^{\perp}-\nabla \theta & =0 \\
\frac{\partial \theta}{\partial t}-\nabla \cdot \mathbf{u} & =\frac{1}{\tau_{\text {low }}} q_{\text {low }}+\frac{1}{\tau_{\text {mid }}} q_{\text {mid }}, \\
\frac{\partial q_{\text {low }}}{\partial t}+\tilde{Q}_{\text {low }} \nabla \cdot \mathbf{u} & =-\frac{1}{\tau_{\text {low }}} q_{\text {low }}, \quad \text { and } \\
\frac{\partial q_{\mathrm{mid}}}{\partial t}+\tilde{Q}_{\mathrm{mid}} \nabla \cdot \mathbf{u} & =-\frac{1}{\tau_{\mathrm{mid}}} q_{\mathrm{mid}},
\end{aligned}
$$

where other additional features (diffusion, etc.) will still be left out until the complete system is considered in section 4c. As before, it will also be useful in what follows to consider an additional equation for a vertically integrated moist static energy,

$$
h=\theta+q_{\mathrm{low}}+q_{\mathrm{mid}},
$$

which evolves according to the expression

$$
\frac{\partial h}{\partial t}-\left(1-\tilde{Q}_{\text {low }}-\tilde{Q}_{\text {mid }}\right) \nabla \cdot \mathbf{u}=0 .
$$

The scaling assumptions in this case are expanded to include assumptions about both lower and middle tropospheric parameters:

$$
\tau_{\text {low }}=\varepsilon, \quad \tau_{\text {mid }}=O(1) \quad \text { and }
$$




$$
1-\tilde{Q}_{\mathrm{low}}=O(\varepsilon), \quad \tilde{Q}_{\mathrm{mid}}=\varepsilon \tilde{Q}_{m}=O(\varepsilon) .
$$

The $\tau_{\text {low }}$ and $\tilde{Q}_{\text {low }}$ scalings are the same as in the onelevel case considered above. By comparison, the midtroposphere convective adjustment time $\tau_{\text {mid }}$ is taken to be larger than $\tau_{\text {low }}$, and the midtroposphere vertical moisture gradient $\tilde{Q}_{\text {mid }}$ is taken to be smaller than $\tilde{Q}_{\text {low }}$. These scalings are mostly in line with the standard parameter values of Stechmann and Hottovy (2017) and (4), except the standard parameter value $\tilde{Q}_{\text {mid }}=0.45$ is somewhat intermediate between $O(1)$ and $O(\varepsilon)$; the choice of $\tilde{Q}_{\text {mid }}=O(\varepsilon)$ leads to more reasonable balance relations below and is consistent with a vertical moisture gradient that decreases significantly with height.

Aside from the additional scaling assumptions above, the remaining steps of the derivation are similar to the one-level case and will be described succinctly as follows. A rescaling of water vapor and time is introduced for convenience,

$$
q_{\mathrm{low}}=\varepsilon q_{l}, \quad q_{\mathrm{mid}}=\varepsilon q_{m}, \quad \text { and } \quad t^{\prime}=\varepsilon t,
$$

and the rescaled equations are then

$$
\begin{aligned}
\varepsilon \frac{\partial \mathbf{u}}{\partial t^{\prime}}+y \mathbf{u}^{\perp}-\nabla \theta & =0, \\
\varepsilon \frac{\partial \theta}{\partial t^{\prime}}-\nabla \cdot \mathbf{u} & =q_{l}+\varepsilon \frac{1}{\tau_{\mathrm{mid}}} q_{m}, \\
\varepsilon^{2} \frac{\partial q_{l}}{\partial t^{\prime}}+\tilde{Q}_{\mathrm{low}} \nabla \cdot \mathbf{u} & =-q_{l}, \quad \text { and } \\
\varepsilon^{2} \frac{\partial q_{m}}{\partial t^{\prime}}+\varepsilon \tilde{Q}_{m} \nabla \cdot \mathbf{u} & =-\varepsilon \frac{1}{\tau_{\mathrm{mid}}} q_{m} .
\end{aligned}
$$

The leading-order balance relations are immediately seen to be

$$
\begin{aligned}
& y \mathbf{u}^{\perp}-\nabla \theta=0, \\
& \tilde{Q}_{\text {low }} \nabla \cdot \mathbf{u}=-\frac{q_{\text {low }}}{\tau_{\text {low }}}, \text { and } \\
& \tilde{Q}_{\text {mid }} \nabla \cdot \mathbf{u}=-\frac{q_{\text {mid }}}{\tau_{\text {mid }}},
\end{aligned}
$$

and the moist static energy equation is

$$
\frac{\partial \theta}{\partial t}-\left(1-\tilde{Q}_{\text {low }}-\tilde{Q}_{\text {mid }}\right) \nabla \cdot \mathbf{u}=0
$$

to leading order.

Last, one can derive the following equation for $q_{\mathrm{mid}}$ alone:

$$
\frac{\partial}{\partial t}\left(y^{2} q_{\text {mid }}\right)+\left(\tilde{Q}_{\text {low }}+\tilde{Q}_{\text {mid }}-1\right) \frac{\partial}{\partial x} q_{\text {mid }}=0
$$

which again looks similar to an advection equation.
To conclude this case, notice the most significant difference here: a propagation velocity of

$$
c \sim \tilde{Q}_{\text {low }}+\tilde{Q}_{\text {mid }}-1
$$

is suggested here, in contrast to $\tilde{Q}-1$ in the one-level case. This change has significant consequences for the propagation direction. With standard value of $\tilde{Q}_{\text {low }}=$ 0.9 , one can see that eastward propagation is now possible if $\tilde{Q}_{\text {mid }}>0.1$. This was made possible through simple convective adjustment mechanisms, if using two vertical levels.

\section{c. Two-vertical-level convective adjustment, with full model setup}

The full system from (4) will now be investigated, and an asymptotic derivation will identify a single, unidirectional wave on planetary and intraseasonal scales. The previous, simplified cases provide useful background and indicate some of the basic asymptotic features, but they deviated from the full system in that they neglected diffusion and other dissipation, and they were based on synoptic scaling for time and space scales. The derivation here will be described succinctly, since it is similar to the earlier simplified cases.

To investigate the full system in (4), the scaling assumptions will be

$$
\begin{aligned}
1-\tilde{Q}_{\mathrm{low}} & =1-\tilde{Q}_{l}=\varepsilon, \quad \tilde{Q}_{\mathrm{mid}}=\varepsilon \tilde{Q}_{m}=O(\varepsilon), \\
\tau_{\mathrm{low}} & =\tau_{l}=O(1), \quad \tau_{\mathrm{mid}}=\varepsilon^{-1} \tau_{m}=O\left(\varepsilon^{-1}\right), \\
b_{\mathrm{low}} & =\varepsilon b_{l}=O(\varepsilon), \quad b_{\mathrm{mid}}=\varepsilon b_{m}=O(\varepsilon), \quad \text { and } \\
\tau_{u} & =\varepsilon^{-2} \tau=O\left(\varepsilon^{-2}\right), \quad \tau_{\theta}=\varepsilon^{-2} \tau=O\left(\varepsilon^{-2}\right) .
\end{aligned}
$$

In brief, the $\tilde{Q}_{\text {low }}$ and $\tilde{Q}_{\text {mid }}$ scalings are the same as in the one-level case considered above, the midtroposphere convective adjustment time $\tau_{\text {mid }}$ is taken to be larger than $\tau_{\text {low }}$, the eddy diffusivities $b_{\text {low }}$ and $b_{\text {mid }}$ are assumed to be small, and the damping time scales $\tau_{u}$ and $\tau_{\theta}$ are assumed to be large. These scalings are mostly in line with the standard parameter values of Stechmann and Hottovy (2017) and (4), except the standard parameter value $\tilde{Q}_{\text {mid }}=0.45$ is somewhat intermediate between $O(1)$ and $O(\varepsilon)$, as discussed earlier; and the standard parameter value $b_{\text {mid }}=0.8$ is perhaps closer to $O(1)$, but a smaller $O(\varepsilon)$ value is somewhat close and provides the most reasonable balance relation in the asymptotic limit, as described below.

A rescaling of water vapor and time is introduced for convenience, as in the earlier simpler cases, as well as additional rescalings for planetary and intraseasonal scaling of $v, x$, and $t$ : 


$$
\begin{aligned}
q_{\text {low }} & =\varepsilon q_{l}, \quad q_{\text {mid }}=\varepsilon q_{m}, \quad v=\varepsilon v^{\prime}, \quad x^{\prime}=\varepsilon x, \quad \text { and } \\
t^{\prime} & =\varepsilon^{2} t,
\end{aligned}
$$

along with the notation

$$
\nabla^{\prime} \cdot \mathbf{u}^{\prime}=\frac{\partial u}{\partial x^{\prime}}+\frac{\partial v^{\prime}}{\partial y},
$$

which involves the long-wave-scaled versions of meridional velocity, $v^{\prime}$, and zonal coordinate, $x^{\prime}$. Note the $\varepsilon^{2}$ factor in the time scale, rather than the scaling of $\varepsilon$ that has been used in other contexts with equatorial longwave scaling (Majda and Klein 2003; Majda and Biello 2004; Biello and Majda 2005). Since $t$ itself represents the synoptic time scale of $\approx 8 \mathrm{~h}$ from dry equatorial wave theory (e.g., Gill 1982a), $\varepsilon^{2} t$ is then a time scale of ( 8 hours) $\times 10^{2} \approx 33$ days, consistent with the MJO. As a comparison from a different perspective, the $\varepsilon t$ time scale (along with the $\varepsilon x$ length scale) is the dry Kelvin wave time scale, associated with a wave speed of $50 \mathrm{~m} \mathrm{~s}^{-1}$. In contrast, the $\varepsilon^{2} t$ time scale is slower than $\varepsilon t$ by a factor of $\varepsilon$; for a value of $\varepsilon \approx 0.1$, it corresponds to a slower wave speed of $5 \mathrm{~m} \mathrm{~s}^{-1}$, consistent with the MJO.

In terms of these rescaled variables, the full model in (4) becomes

$$
\begin{aligned}
\varepsilon^{2} \frac{\partial u}{\partial t^{\prime}}-\varepsilon y v^{\prime}-\varepsilon \frac{\partial \theta}{\partial x^{\prime}} & =-\varepsilon^{2} \frac{1}{\tau} u, \\
\varepsilon^{3} \frac{\partial v^{\prime}}{\partial t^{\prime}}+y u-\frac{\partial \theta}{\partial y} & =-\varepsilon^{3} \frac{1}{\tau} v^{\prime}, \\
\varepsilon^{2} \frac{\partial \theta}{\partial t^{\prime}}-\varepsilon \nabla^{\prime} \cdot \mathbf{u}^{\prime} & =\varepsilon \frac{1}{\tau_{l}} q_{l}+\varepsilon^{2} \frac{1}{\tau_{m}} q_{m}-\varepsilon^{2} \frac{1}{\tau} \theta, \\
\varepsilon^{3} \frac{\partial q_{l}}{\partial t^{\prime}}+\varepsilon \tilde{Q}_{l} \nabla^{\prime} \cdot \mathbf{u}^{\prime}= & -\varepsilon \frac{1}{\tau_{l}} q_{l}+\varepsilon^{4} b_{l} \partial_{x^{\prime}}^{2} q_{l} \\
& +\varepsilon^{2} b_{l} \partial_{y}^{2} q_{l}, \text { and }
\end{aligned}
$$

$$
\begin{aligned}
\varepsilon^{3} \frac{\partial q_{m}}{\partial t^{\prime}}+\varepsilon^{2} \tilde{Q}_{m} \nabla^{\prime} \cdot \mathbf{u}^{\prime}= & -\varepsilon^{2} \frac{1}{\tau_{m}} q_{m}+\varepsilon^{4} b_{m} \partial_{x \prime}^{2} q_{m} \\
& +\varepsilon^{2} b_{m} \partial_{y}^{2} q_{m} .
\end{aligned}
$$

The leading-order balance relations are immediately seen to be

$$
\begin{aligned}
& y \mathbf{u}^{\perp}-\nabla \theta=0, \\
& \tilde{Q}_{\text {low }} \nabla \cdot \mathbf{u}=-\frac{1}{\tau_{\text {low }}} q_{\text {low }}, \quad \text { and } \\
& \tilde{Q}_{\text {mid }} \nabla \cdot \mathbf{u}=-\frac{1}{\tau_{\text {mid }}} q_{\text {mid }}+b_{\text {mid }} \partial_{y}^{2} q_{\text {mid }} .
\end{aligned}
$$

[Note that the balance in, e.g., (33c) was seen as the leading-order balances in the budget analysis in section 3 and Table 2.] The moist static energy evolution in this case is found by adding (32c)-(32e) (see the online supplemental material for more detail) to obtain

$$
\begin{aligned}
\frac{\partial \theta}{\partial t}-\left(1-\tilde{Q}_{\text {low }}-\tilde{Q}_{\text {mid }}\right) \nabla \cdot \mathbf{u}= & -\frac{1}{\tau_{\theta}} \theta+b_{\text {low }} \partial_{y}^{2} q_{\text {low }} \\
& +b_{\text {mid }} \partial_{y}^{2} q_{\text {mid }},
\end{aligned}
$$

which arises as the leading-order equation for the dynamics of $h=\theta+q_{\text {low }}+q_{\text {mid }} \approx \theta$. Note that the original scaling for all variables $(x, t, v$, etc.) has been used again, for simplicity in notation, and therefore it must be implicitly understood that these equations are valid only on planetary zonal scales, and so on, as described earlier in the scaling assumptions. The main difference here, in comparison with the earlier, simplified case of section $4 b$, is the appearance of Newtonian cooling, $-\theta / \tau_{\theta}$, and meridional eddy diffusion, $\partial_{y}^{2} q_{\text {low }}$ and $\partial_{y}^{2} q_{\text {mid }}$.

A single propagating wave can be revealed by rewriting (33) as a single equation for $q_{\text {mid }}$ alone. Following the same type of procedure as in sections $4 \mathrm{a}$ and $4 \mathrm{~b}$ leads to

$$
\begin{gathered}
\frac{\partial}{\partial t}\left[y^{2}\left(1-\tau_{\text {mid }} b_{\text {mid }} \partial_{y}^{2}\right) q_{\text {mid }}\right]+\left(\tilde{Q}_{\text {low }}+\tilde{Q}_{\text {mid }}-1\right) \frac{\partial}{\partial x}\left[\left(1-\tau_{\text {mid }} b_{\text {mid }} \partial_{y}^{2}\right) q_{\text {mid }}\right]+\left(\tau_{\text {mid }} \tilde{Q}_{\text {mid }} b_{\text {mid }}+\tau_{\text {low }} \tilde{Q}_{\text {low }} b_{\text {low }}\right) \frac{\partial}{\partial x}\left(\partial_{y}^{2} q_{\text {mid }}\right) \\
-\tau_{\text {mid }} \tau_{\text {low }} \tilde{Q}_{\text {low }} b_{\text {low }} b_{\text {mid }} \frac{\partial}{\partial x}\left(\partial_{y}^{4} q_{\text {mid }}\right)+\tau_{\text {mid }} \tilde{Q}_{\text {mid }} b_{\text {mid }} \frac{\partial^{3}}{\partial x^{3}} q_{\text {mid }}=-\frac{1}{\tau_{\theta}}\left[y^{2}\left(1-\tau_{\text {mid }} b_{\text {mid }} \partial_{y}^{2}\right) q_{\text {mid }}\right] .
\end{gathered}
$$

Note that zonal diffusion of $q_{\text {mid }}$ was included here and leads to the $\partial^{3} / \partial x^{3}$ term; while it will be asymptotically negligible at the largest planetary scales, it was added here because it can have an appreciable impact for some zonal wavenumbers and is therefore interesting to include. Moreover, notice that zonal diffusion, a dissipation mechanism, actually leads to a dispersive effect, as indicated by the order-three derivative, $\partial^{3} / \partial x^{3}$. If all diffusion and dissipation terms are neglected by setting $b_{\text {mid }}=b_{\text {low }}=\tau_{\theta}^{-1}=0$, then notice that the earlier 

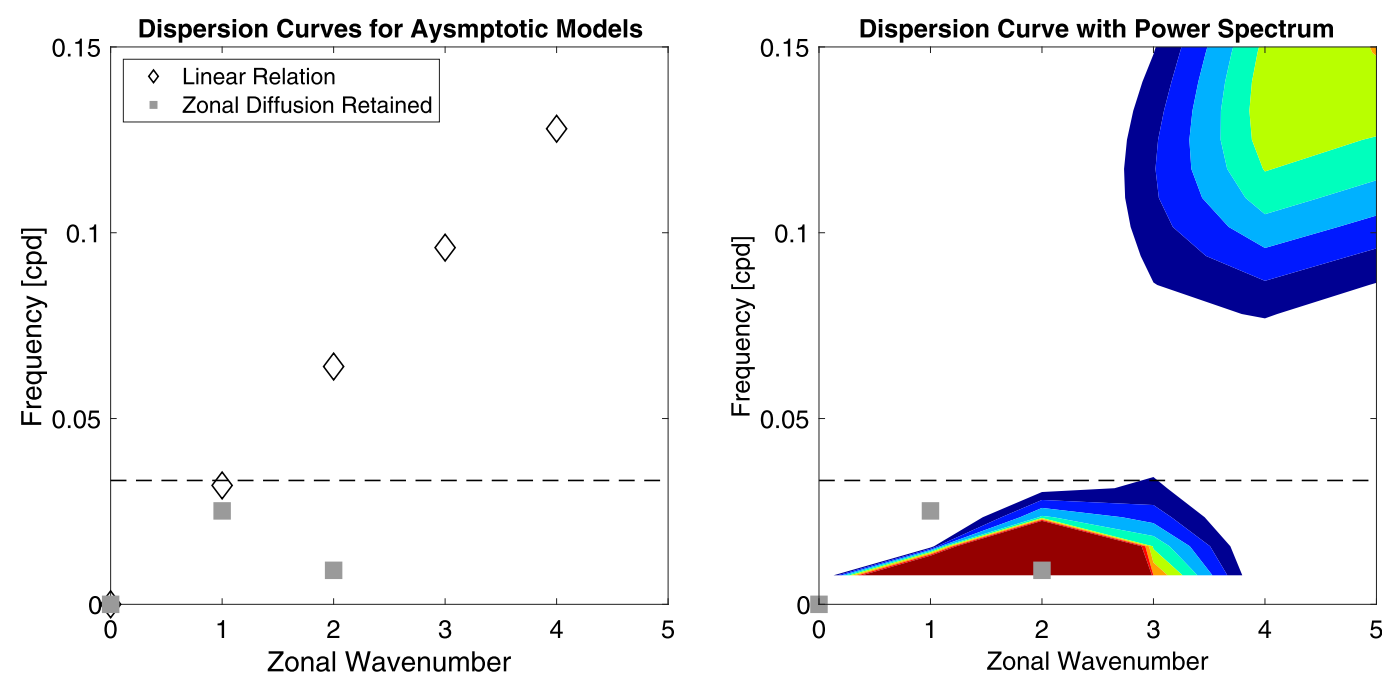

FIG. 8. The dispersion curves for the asymptotic system of (33). The system was expanded into meridional basis functions and truncated to retain up to $\phi_{2}$ for the moisture terms. Shown are (left) the linear relationship (diamonds) and when zonal diffusion is retained (squares) and (right) the same dispersion relationship with the power spectrum of the full model, system (4).

simplified result from (27) is obtained; hence diffusion and radiation can add some further features on top of the basic mechanism of slow propagation related to $\tilde{Q}_{\text {low }}+\tilde{Q}_{\text {mid }}-1$.

To distill the basic aspects of (34), one can derive a version of it that utilizes simplified meridional basis functions, such as $q_{\text {low }}(x, y, t) \propto q_{\text {low }, 0}(x, t) \exp \left(-y^{2} / 2\right)$. By applying such simplifications to (33) and using properties of the meridional basis functions (e.g., Majda 2003; Stechmann and Hottovy 2017), one can obtain

$$
\frac{\partial}{\partial t} q_{\mathrm{mid}, 0}+A \frac{\partial}{\partial x} q_{\mathrm{mid}, 0}+B \frac{\partial^{3}}{\partial x^{3}} q_{\mathrm{mid}, 0}=-C q_{\mathrm{mid}, 0},
$$

where the constant coefficients are given by $A=0.2909$, $B=1.0132$, and $C=0.04$. An outline of the calculation for these constants is given in the online supplemental material. The use of meridional basis functions has removed the appearance of $y$ and led to a PDE in terms of the zonal coordinate $x$ alone. The dispersion relationship for the PDE is

$$
\omega=A k-B k^{3}-i C
$$

This PDE represents a propagating wave, and its dispersion curve is shown in Fig. 8 (diamonds for the $A$ term, and squares for the $A$ and $B$ term included). The dispersion curve is roughly aligned with the MJO's spectral peak, indicating that the asymptotic wave is roughly in agreement with the MJO of the full model.

In brief, in looking back at the hierarchy of model versions in sections $4 a, 4 b$, and $4 c$, one can see the basic features arising in the simplest versions: the structure of the intraseasonal oscillations is in the form of tropical geostrophic balance, and the propagation is influenced by vertical gradients of background moisture.

\section{Discussion}

Two additional topics are discussed in this section: more general convective adjustment schemes (section 5a), and nonlinear advection of moist static energy, and additional processes (section $5 b$ ).

\section{a. More general convective adjustment schemes}

In section 4, convective adjustment was used in the form of $P=q / \tau$. This is a linear formulation, and no convective trigger is used (or, in other words, the convective trigger is always assumed to be turned on), with the result that moisture anomalies were constrained, by convective adjustment, to be relatively small. The result of small moisture can be removed, as demonstrated in several ways in this section, by moving beyond the simplest version of convective adjustment and adding further complexity to the models.

\section{1) NONLINEAR THRESHOLD FOR CONVECTIVE TRIGGER}

To include a convective trigger, one could consider replacing the linear relation $P=q / \tau$ and instead using

$$
P=q^{+} / \tau
$$


where the superscript plus sign is shorthand notation for the ramp function: $q^{+}=\max (q, 0)$. Hence, the convective trigger is turned on only when $q$ exceeds the threshold value of $q=0$.

If the convective adjustment time scale $\tau$ is relatively fast, then the leading-order condition would be

$$
q^{+} / \tau=0 .
$$

A similar (but linear) condition of $q / \tau=0$ arose in section 4 and resulted in small moisture; here in (38), with $q_{0}^{+} / \tau=0$, it is seen that moisture anomalies are constrained to be small only when the convective trigger is turned on-that is, only when $q \geq 0$. [Even this constraint can be removed by allowing the convective threshold to depend on temperature; see section 5a(2)].

\section{2) CONVECTIVE THRESHOLD WITH TEMPERATURE DEPENDENCE}

In (37), the threshold for convection was taken to be the constant value $q=0$, for simplicity. Further realism could come from allowing a convective threshold $q_{c}$ that depends on temperature, in which case (37) becomes

$$
P=\left[q-q_{c}(\theta)\right]^{+} / \tau .
$$

For instance, one could use $q_{c}(\theta)=\alpha \theta$, where $\alpha$ is a constant. Such a choice has some observational support; for example, Neelin et al. (2009) examine the critical water vapor value for the onset of deep convection and find that it is temperature dependent and roughly a linear function of temperature. If (39) is used, and if the convective adjustment time scale $\tau$ is relatively fast, then the leading-order condition would no longer be (38); instead, it would be $\left[q-q_{c}(\theta)\right]^{+} / \tau=0$. Hence, moisture anomalies are not constrained to be small. Instead, moisture anomalies are constrained to be related to temperature anomalies, at least when the convective trigger is turned on. Some observational evidence can be seen, in the troposphere, for temperature anomalies to be nearly in phase with precipitation and water vapor anomalies, for the MJO, to a leading-order approximation (see, e.g., Kiladis et al. 2005; Benedict and Randall 2007). Therefore, by using a threshold $q_{c}(\theta)$ instead of a constant threshold $q_{c}=0$, the result of small water vapor anomalies is removed.

\section{3) LONGER CONVECTIVE ADJUSTMENT TIME}

As yet another way to allow larger moisture anomalies, one can use a longer convective adjustment time. As an illustration, suppose that both moisture $q$ and the convective adjustment time scale $\tau$ are moderate and $O(1)$ (instead of being small), and the rest of the setup is identical to section 4a. Then the results of section $4 \mathrm{a}$ are all essentially the same, except the water vapor $q$ is not small, and therefore the moist static energy is $h=\theta+q$. The final result in (13) is identical, except $\partial \theta / \partial t$ should be $\partial h / \partial t$. Hence the water vapor $q$ is allowed to make a substantial contribution to the moist static energy $h$, and it is possible even for $q$ to make a larger contribution than $\theta$, or vice versa, depending on the details of the structure.

In fact, the structure is also the same, and it reveals the relationship between $\theta$ and $q$. The structure is determined from the leading-order balance relations,

$$
\begin{aligned}
y \mathbf{u}^{\perp}-\nabla \theta & =0 \quad \text { and } \\
\tilde{Q} \nabla \cdot \mathbf{u} & =-\frac{1}{\tau} q,
\end{aligned}
$$

which are identical whether one assumes fast $O(\varepsilon) \tau$ or moderate $O(1) \tau$, since $q$ scales accordingly to maintain the same ratio $q / \tau$. Consequently, for this case of a longer convective adjustment time, the structure is still given by Fig. 7. This structure in the figure comes from the balance relation in (18a),

$$
\partial_{x} \theta=-y^{2} \frac{q}{\tilde{Q} \tau} .
$$

One can see that $\theta$ is proportional to $y^{2}$ and is therefore small near the equator. The moist static energy near the equator is therefore dominated by contributions from moisture $q$.

In analyses of MJO data from reanalysis and global climate models (GCMs), moist static energy anomalies have been seen to be dominated by moisture anomalies, near the equator (e.g., Maloney 2009; Kiranmayi and Maloney 2011). The result in (41) is a balance relation that essentially demonstrates the same behavior. Namely, $\theta$ anomalies are smaller than $q$ anomalies, near the equator. This behavior is a property of the Matsuno-Gill-like system, without damping, in (40).

Note that, in this case of assuming $\tau$ to be moderate and $O(1)$, it is advantageous to find that moist static energy is moisture dominated; nevertheless, for moderate $\tau$, the simplified CCEW model in (2) does not appear to arise. Such a lack of a corresponding simplified CCEW model (at least in a one-vertical-mode setup) is perhaps a disadvantage of the moderate $\tau$ case. The possibility of having simplified models for both CCEWs and the MJO is one motivating factor for considering the case of fast $\tau$ in section 4 a.

\section{b. Nonlinear advection of moist static energy and additional processes}

A number of other processes are also important to consider. For instance, as background, some studies have examined the moisture and moist static energy budget of the 
MJO in observational data, reanalysis data, and/or climate model simulation data (e.g., Benedict and Randall 2007; Maloney 2009; Kiranmayi and Maloney 2011; Andersen and Kuang 2012; Sobel et al. 2014; Inoue and Back 2015a). Several of these studies emphasize longwave cloud-radiation feedbacks and horizontal moisture advection as dominant processes for the maintenance and propagation of the observed MJO, respectively. The purpose of this subsection is to further generalize the models to show how these additional processes fit within the present framework.

Note that horizontal moisture advection was already included to an extent in the earlier sections 2 and 4. One could break moisture advection into two componentsplanetary-scale advection versus advection by eddies - and it is the (meridional) advection by synoptic-scale eddies that has been seen to dominate over planetary-scale advection (e.g., Maloney 2009; Kiranmayi and Maloney 2011; Andersen and Kuang 2012). Here, in earlier sections 2 and 4 , advection of moisture by eddies was included in the model in the form of eddy diffusion. The other component-planetary-scale advection - is difficult or impossible to include in a model whose vertical structure uses only the first baroclinic mode, without a barotropic mode or other vertical structure (e.g., Majda and Biello 2003; Chen et al. 2015, 2016); so planetary-scale advection was not included in the earlier sections 2 and 4, but it can be included in a model with full vertical structure, as will be shown now.

As a three-dimensional setup with nonlinear advection, the starting point will be the three-dimensional primitive equations, in nondimensional units:

$$
\begin{aligned}
\frac{D \mathbf{U}}{D t}+y \mathbf{U}^{\perp} & =-\nabla P \\
\nabla \cdot \mathbf{U}+\frac{\partial W}{\partial z} & =0 \\
\frac{\partial P}{\partial z} & =\Theta \\
\frac{D \Theta}{D t}+W & =S^{\Theta} \\
\frac{D Q}{D t}-\tilde{Q} W & =S^{Q},
\end{aligned}
$$

This setup follows the supporting information of Stechmann and Hottovy (2017) and uses nondimensional units that are the same as in Stechmann and Majda (2015). The material derivative is

$$
\frac{D}{D t}=\frac{\partial}{\partial t}+\mathbf{U} \cdot \nabla+W \frac{\partial}{\partial z}
$$

Here the horizonal velocity is $\mathbf{U}=[U(x, y, z, t), V(x, y, z$, $t)$ ], and $\nabla$ is the horizonal gradient operator: $\mathbf{U} \cdot \nabla=$ $U \partial_{x}+V \partial_{y}$. The vertical velocity is $W$, and the pressure is
$P$. The term $y \mathbf{U}^{\perp}$ represents the Coriolis force, under the equatorial beta-plane approximation, and where $\mathbf{U}^{\perp}=$ $(-V, U)$. The potential temperature $\Theta$ and water vapor mixing ratio $Q$ are anomalies from background states that are linear with respect to height $z$ :

$$
\begin{aligned}
& \theta^{\mathrm{tot}}=\theta_{00}+z+\Theta(x, y, z, t) \quad \text { and } \\
& q^{\mathrm{tot}}=q_{00}-\tilde{Q} z+Q(x, y, z, t),
\end{aligned}
$$

where $\theta_{00}$ and $q_{00}$ are constants and represent values at the top of the boundary layer at $z=0$. For a model of this complexity, it is common to consider the fluid as bounded above and below by rigid lids at the bottom of the troposphere $(z=0)$ and the top of the troposphere ( $z=\pi$ in nondimensional units). The boundary condition at $z=0$ and $z=\pi$ is $W=0$.

To begin the asymptotic analysis, the variables are assumed to be small, in accordance with an assumption of small Froude number. For instance, typical horizontal velocities are assumed to be roughly $5 \mathrm{~m} \mathrm{~s}^{-1}$, which is small relative to the gravity wave speed of $50 \mathrm{~m} \mathrm{~s}^{-1}$. To incorporate this scaling, the variables are rescaled by a factor of $\varepsilon$. That is,

$$
\begin{aligned}
& \mathbf{U}^{\prime}=\mathbf{U} / \varepsilon, \quad P^{\prime}=P / \varepsilon, \quad W^{\prime}=W / \varepsilon, \\
& \Theta^{\prime}=\Theta / \varepsilon, \quad Q^{\prime}=Q / \varepsilon, \quad \text { and } \\
& S^{\Theta \prime}=S^{\Theta} / \varepsilon, \quad S^{Q^{\prime}}=S^{Q} / \varepsilon .
\end{aligned}
$$

Also, the time scale is assumed to be long (i.e., not the gravity wave time scale) and is accordingly rescaled by a factor of $\varepsilon$ :

$$
t^{\prime}=\varepsilon t
$$

With this rescaling, the equations of (42) become

$$
\begin{aligned}
\varepsilon^{2} \frac{D \mathbf{U}}{D t}+\varepsilon y \mathbf{U}^{\perp} & =-\varepsilon \nabla P \\
\varepsilon \nabla \cdot \mathbf{U}+\varepsilon \frac{\partial W}{\partial z} & =0 \\
\varepsilon \frac{\partial P}{\partial z} & =\varepsilon \Theta \\
\varepsilon^{2} \frac{D \Theta}{D t}+\varepsilon W & =\varepsilon S^{\Theta} \\
\varepsilon^{2} \frac{D Q}{D t}-\varepsilon \tilde{Q} W & =\varepsilon S^{Q},
\end{aligned}
$$

and the corresponding equation for the moist static energy $(H=\Theta+Q)$ evolution, in rescaled form, is

$$
\varepsilon^{2} \frac{D H}{D t}+\varepsilon(1-\tilde{Q}) W=\varepsilon\left(S^{\Theta}+S^{Q}\right)
$$


where the primes have been dropped in (46) and (47) to ease notation.

The leading-order balances of (46) are

$$
\begin{aligned}
y \mathbf{U}^{\perp} & =-\nabla P, \\
\nabla \cdot \mathbf{U}+\frac{\partial W}{\partial z} & =0, \\
\frac{\partial P}{\partial z} & =\Theta, \\
W & =S^{\Theta}, \quad \text { and } \\
-\tilde{Q} W & =S^{Q} .
\end{aligned}
$$

These balances can be viewed as the three-dimensional counterparts of the leading-order balances in (12), (26), and (33). To find the leading-order moist static energy equation, from $(47)$, one uses that $(1-\tilde{Q})=O(\varepsilon)$ and $S^{\Theta}+S^{Q}=O(\varepsilon)$, or that, respectively, the moist stability is weak and the heat source and moisture sink are nearly in balance; as a result, (47) becomes, at leading order,

$$
\frac{\partial H}{\partial t}+\mathbf{U} \cdot \nabla H+W \frac{\partial H}{\partial z}+(1-\tilde{Q}) W=S^{\Theta}+S^{Q},
$$

where the material derivative has been written explicitly to show the nonlinearities, and the rescaling by $\varepsilon$ has been removed to restore the original nondimensional form of the equation. This moist static energy evolution equation is actually identical to the full moist static energy evolution equation, without consideration of any scaling assumptions. Nevertheless, performing the scale analysis allows one to see that all of the terms in the moist static energy evolution equation are the same order of magnitude (if the sources are considered together as the sum $S^{\Theta}+S^{Q}$ ). Thus, while nonlinear advection is not leading-order for the heat and moisture balances in (48), it is leading-order for the moist static energy evolution.

Some further remarks are in order. First, notice that additional effects, such as longwave cloud-radiation feedbacks or WISHE, could be included through the heat and moisture sources, $S^{\Theta}$ and $S^{Q}$, and they would appear in the moist static energy evolution at leading order. They were neglected, along with nonlinear advection, in earlier sections in order to emphasize the more basic effects, such as convective adjustment and moist stability, in the simplest way. Also, while planetary-scale advection was neglected in earlier sections to allow a linear analysis, eddy advection was included, in the form of eddy diffusion. Some motivation for considering radiative effects and WISHE comes from the results of Khairoutdinov and Emanuel (2018), who found that the MJO disappears if there are no radiative feedbacks and the MJO does not propagate if there is no WISHE, in a cloud-system-permitting model with grid spacing of $20 \mathrm{~km}$. Second, note that a more comprehensive analysis would use the equatorial longwave scaling of section $4 \mathrm{c}$, but the simpler presentation of this section demonstrates essentially the same ideas without the complications of longwave scaling.

Third, it is interesting to consider this asymptotic limit in the context of the various versions of the WTG and weak buoyancy gradient (WBG) approximations (e.g., Charney 1963; Sobel et al. 2001; Majda and Klein 2003; Stechmann and Stevens 2010; Yang 2018). An important aspect of this paper is the consideration of moist dynamics, so that two thermodynamic variables are represented $(\Theta$ and $Q)$, and a corresponding consideration of two vertical gradients, the potential temperature gradient and the moisture gradient, written here in nondimensional form as 1 and $-\tilde{Q}$, respectively. Both gradients are assumed to be relatively strong, leading to balance equations $W=S^{\theta}$ and $-\tilde{Q} W=S^{Q}$, respectively. An additional important scaling assumption here is that $1-\tilde{Q}$ is relatively small; as a result, $W=S^{\theta}$ and $-\tilde{Q} W=S^{Q}$ represent essentially the same balance, with $S^{\theta}+S^{Q}$ small, and the moist static energy evolution is needed to close the system. It is also interesting to compare our setup with a similar setup in the precipitating quasigeostrophic (PQG) limit of Smith and Stechmann (2017). Both involve small Rossby numbers, as indicated by geostrophic balance. Both also involve small Froude numbers, including two Froude numbers-one dry, one moist. One difference is that, in the PQG setting, the background gradient $d \tilde{\theta}_{e} / d z$ is strong, whereas here the background gradient $d \tilde{\theta}_{e} / d z \sim 1-\tilde{Q}$ is weak. It would be interesting to compare with the PQG-scale analysis or a planetary-geostrophic-scale analysis more thoroughly in the future.

\section{Conclusions}

One of the main goals here was to investigate a simple theory for the MJO's propagation and structure, in a way that is similar to convective adjustment theory for CCEW propagation and structure. Under such a setup, the MJO structure was shown to asymptotically obey a type of tropical geostrophic balance (section 4):

$$
\begin{aligned}
y \mathbf{u}^{\perp}-\nabla \theta & =0 \quad \text { and } \\
\tilde{Q} \nabla \cdot \mathbf{u} & =-\frac{1}{\tau} q .
\end{aligned}
$$


Slow propagation of roughly $5 \mathrm{~m} \mathrm{~s}^{-1}$ was shown to arise from a weak moist stability, $1-\tilde{Q}$, that has a small magnitude of $O(\varepsilon)$ :

$$
c_{\mathrm{MJO}} \propto \tilde{Q}-1 \quad \text { or } \quad c_{\mathrm{MJO}} \propto \tilde{Q}_{\mathrm{low}}+\tilde{Q}_{\mathrm{mid}}-1 .
$$

This basic $c_{\mathrm{MJO}}$ propagation mechanism could be either westward or eastward, depending on the sign of $\tilde{Q}-1$ or $\tilde{Q}_{\text {low }}+\tilde{Q}_{\text {mid }}-1$. For the standard parameter values of section 3 and $4 \mathrm{~b}$,c, the sign of $\tilde{Q}_{\text {low }}+\tilde{Q}_{\text {mid }}-1$ is positive, and MJO propagation is eastward.

In comparing convective adjustment theory for CCEWs and the MJO, there are a few noteworthy points of distinction. First, in terms of propagation, it is the moist stability, $1-\tilde{Q}$, that arises as a key quantity in both cases, but the dependence is different for CCEWs versus the MJO:

$$
c_{\mathrm{CCEW}}=\sqrt{1-\tilde{Q}} \quad \text { vs. } \quad c_{\mathrm{MJO}} \propto 1-\tilde{Q},
$$

with a square root dependence for CCEWs [i.e., (3)] and a linear dependence for the MJO. For a representative value of $1-\tilde{Q}=0.1$, these speeds correspond to values of

$$
c_{\mathrm{CCEW}} \sim 15 \mathrm{~m} \mathrm{~s}^{-1} \quad \text { vs. } \quad c_{\mathrm{MJO}} \sim 5 \mathrm{~m} \mathrm{~s}^{-1},
$$

where a reference velocity scale of $50 \mathrm{~m} \mathrm{~s}^{-1}$ had been used for nondimensionalization. Both of these values are reasonable estimates, yet they arise from different scaling assumptions. In particular, for CCEWs, no asymptotic smallness is assumed for $1-\tilde{Q}$, whereas for the MJO here, it was assumed that $1-\tilde{Q}=O(\varepsilon)$-that is, that the moist stability is nearly neutral. The former assumption leads to a complete set of equatorial waves from shallow water theory, whereas the latter assumption leads to a single, slow, unidirectional wave. Second, in terms of structure, the CCEWs have the same structures as their counterparts from dry shallow water theory, except the meridional length scales are reduced by a factor of $(1-\tilde{Q})^{1 / 2}$ due to a reduced, moist stability. For the MJO's structure, on the other hand, there is no such reduction in meridional length scale.

Solving the asymptotic model is easy, for the asymptotic MJO structure. Given the moisture $q$ or the precipitation $-q / \tau$, the system for tropical geostrophic balance in (50) can be solved for $u, v$, and $\theta$ using simple formulas; see (18). The result is the asymptotic MJO structure shown in Fig. 7. It is easier to solve than the steady models of Matsuno (1966) and Gill (1980), which are more difficult to solve due to their damping terms and are typically solved using equatorial wave decompositions. For the propagation in the asymptotic MJO model, however, it was helpful to use decompositions in terms of meridional basis functions. In particular, the advection-like equations include a $y^{2}$ factor, and it is convenient to interpret them using parabolic cylinder function expansions; see, for example, (17), (27), and (34) and (35). Alternatively, one could view the propagation from its origin: the evolution of the moist static energy; see, for example, (8), (22), and (33d).

The use of a linear model allows many simplifications, and some limitations will naturally also be present. For linear versions of convective adjustment, with relatively fast adjustment time, one limitation is the prediction of small moisture anomalies. This occurs not only for the MJO (section 4) but also in traditional convective adjustment theory for CCEWs (e.g., Gill 1982b; Neelin and Yu 1994; Frierson et al. 2004; Ogrosky and Stechmann 2016). In any case, the small moisture arises only in the simplest case, and it can be removed by many types of slight generalizations of the convective parameterization (see section 5), such as adding a nonlinear threshold or using a moderate value of adjustment time $\tau$. In fact, it follows from the balance relations in (50) that $\partial_{x} \theta=-y^{2} q /(\tilde{Q} \tau)$, so near the equator where $y$ is small, if a moderate $\tau$ value is used, the temperature will actually be smaller than the moisture $q$, and moisture $q$ will make the largest contribution to moist static energy.

The asymptotic limit of convective adjustment theory offers simple predictions of propagation and structure, but it does not address other aspects of CCEW or MJO behavior. For example, aspects such as growth/ decay, scale selection, and so on are seen instead from the model with finite-valued coefficients instead of an asymptotic limit. Budget studies were presented here (section 3) to describe the model processes involved in these other aspects. For instance, scale selection was seen to be influenced by eddy diffusion of moisture (a parameterization of moisture advection by eddies) and by a change in the MJO eigenmode to be more Rossby wave-like than Kelvin wave-like on smaller length scales.

Acknowledgments. The authors thank Chidong Zhang and three anonymous reviewers for helpful discussion. The research of author Stechmann is partially supported by a Sloan Research Fellowship from the Alfred P. Sloan Foundation and a Vilas Associates Award from the University of Wisconsin-Madison. The research of author Hottovy is partially supported by the National Science Foundation under Grant DMS-1815061. 


\section{REFERENCES}

Adames, Á. F., and D. Kim, 2016: The MJO as a dispersive, convectively coupled moisture wave: Theory and observations. J. Atmos. Sci., 73, 913-941, https://doi.org/10.1175/JAS-D-150170.1.

Andersen, J. A., and Z. Kuang, 2012: Moist static energy budget of MJO-like disturbances in the atmosphere of a zonally symmetric aquaplanet. J. Climate, 25, 2782-2804, https://doi.org/ 10.1175/JCLI-D-11-00168.1.

Benedict, J. J., and D. A. Randall, 2007: Observed characteristics of the MJO relative to maximum rainfall. J. Atmos. Sci., 64, 2332-2354, https://doi.org/10.1175/JAS3968.1.

Biello, J. A., and A. J. Majda, 2005: A new multiscale model for the Madden-Julian oscillation. J. Atmos. Sci., 62, 1694-1721, https://doi.org/10.1175/JAS3455.1.

Bretherton, C. S., and P. K. Smolarkiewicz, 1989: Gravity waves, compensating subsidence and detrainment around cumulus clouds. J. Atmos. Sci., 46, 740-759, https://doi.org/10.1175/ 1520-0469(1989)046<0740:GWCSAD > 2.0.CO;2.

Charney, J. G., 1963: A note on large-scale motions in the tropics. J. Atmos. Sci., 20, 607-609, https://doi.org/10.1175/1520 0469(1963)020<0607:ANOLSM > 2.0.CO;2.

Chen, S., A. J. Majda, and S. N. Stechmann, 2015: Multiscale asymptotics for the skeleton of the Madden-Julian oscillation and tropical-extratropical interactions. Math. Climate Wea. Forecasting, 1, 43-69, https://doi.org/10.1515/MCWF2015-0003.

,-- , and — 2016: Tropical-extratropical interactions with the MJO skeleton and climatological mean flow. J. Atmos. Sci., 73, 4101-4116, https://doi.org/10.1175/JAS-D-16-0041.1.

Deng, Q., B. Khouider, and A. J. Majda, 2015: The MJO in a coarse-resolution GCM with a stochastic multicloud parameterization. J. Atmos. Sci., 72, 55-74, https://doi.org/10.1175/ JAS-D-14-0120.1.

Emanuel, K. A., 1987: An air-sea interaction model of intraseasonal oscillations in the tropics. J. Atmos. Sci., 44, 2324-2340, https://doi.org/10.1175/1520-0469(1987)044<2324:AASIMO> 2.0.CO;2

Frierson, D. M. W., A. J. Majda, and O. M. Pauluis, 2004: Large scale dynamics of precipitation fronts in the tropical atmosphere: A novel relaxation limit. Commun. Math. Sci., 2, 591626, https://doi.org/10.4310/CMS.2004.v2.n4.a3.

Gill, A. E., 1980: Some simple solutions for heat-induced tropical circulation. Quart. J. Roy. Meteor. Soc., 106, 447-462, https:// doi.org/10.1002/qj.49710644905.

_ 1982a: Atmosphere-Ocean Dynamics. International Geophysics Series, Vol. 30, Academic Press, 662 pp.

_ $1982 \mathrm{~b}$ : Studies of moisture effects in simple atmospheric models: The stable case. Geophys. Astrophys. Fluid Dyn., 19 119-152, https://doi.org/10.1080/03091928208208950.

Hendon, H. H., and M. L. Salby, 1994: The life cycle of the Madden-Julian oscillation. J. Atmos. Sci., 51, 2225-2237, https://doi.org/10.1175/1520-0469(1994)051<2225:TLCOTM> 2.0.CO;2.

— M. C. Wheeler, and C. Zhang, 2007: Seasonal dependence of the MJO-ENS relationship. J. Climate, 20, 531-543, https:// doi.org/10.1175/JCLI4003.1.

Holloway, C. E., and J. D. Neelin, 2009: Moisture vertical structure, column water vapor, and tropical deep convection. J. Atmos. Sci., 66, 1665-1683, https://doi.org/10.1175/2008JAS2806.1.

Hottovy, S. A., and S. N. Stechmann, 2015: A spatiotemporal stochastic model for tropical precipitation and water vapor dy- namics. J. Atmos. Sci., 72, 4721-4738, https://doi.org/10.1175/ JAS-D-15-0119.1.

Inoue, K., and L. Back, 2015a: Column-integrated moist static energy budget analysis on various time scales during TOGA COARE. J. Atmos. Sci., 72, 1856-1871, https://doi.org/ 10.1175/JAS-D-14-0249.1.

, and _ 2015b: Gross moist stability assessment during TOGA COARE: Various interpretations of gross moist stability. J. Atmos. Sci., 72, 4148-4166, https://doi.org/10.1175/ JAS-D-15-0092.1.

Jiang, X., M. Zhao, E. D. Maloney, and D. E. Waliser, 2016: Convective moisture adjustment time scale as a key factor in regulating model amplitude of the Madden-Julian oscillation. Geophys. Res. Lett., 43, 10 412-10 419, https://doi.org/10.1002/ 2016GL070898.

Jones, C., 2000: Occurrence of extreme precipitation events in California and relationships with the Madden-Julian oscillation. J. Climate, 13, 3576-3587, https://doi.org/10.1175/15200442(2000)013<3576:OOEPEI >2.0.CO;2.

_ L. M. V. Carvalho, R. Wayne Higgins, D. E. Waliser, and J. K. E. Schemm, 2004: A statistical forecast model of tropical intraseasonal convective anomalies. J. Climate, 17, 2078-2095, https://doi.org/10.1175/1520-0442(2004)017<2078:ASFMOT> 2.0. $\mathrm{CO} ; 2$

Khairoutdinov, M. F., and K. Emanuel, 2018: Intraseasonal variability in a cloud-permitting near-global equatorial aquaplanet model. J. Atmos. Sci., 75, 4337-4355, https://doi.org/ 10.1175/JAS-D-18-0152.1.

Khouider, B., and A. J. Majda, 2006: A simple multicloud parameterization for convectively coupled tropical waves. Part I: Linear analysis. J. Atmos. Sci., 63, 1308-1323, https://doi.org/ 10.1175/JAS3677.1.

— A. St-Cyr, A. J. Majda, and J. Tribbia, 2011: The MJO and convectively coupled waves in a coarse-resolution GCM with a simple multicloud parameterization. J. Atmos. Sci., 68, 240-264, https://doi.org/10.1175/2010JAS3443.1.

Kiladis, G. N., K. H. Straub, and P. T. Haertel, 2005: Zonal and vertical structure of the Madden-Julian oscillation. J. Atmos. Sci., 62, 2790-2809, https://doi.org/10.1175/JAS3520.1.

, M. C. Wheeler, P. T. Haertel, K. H. Straub, and P. E. Roundy, 2009: Convectively coupled equatorial waves. Rev. Geophys., 47, RG2003, https://doi.org/10.1029/2008RG000266.

Kiranmayi, L., and E. D. Maloney, 2011: Intraseasonal moist static energy budget in reanalysis data. J. Geophys. Res., 116, D21117, https://doi.org/10.1029/2011JD016031.

Klingaman, N. P., X. Jiang, P. K. Xavier, J. Petch, D. Waliser, and S. J. Woolnough, 2015: Vertical structure and physical processes of the Madden-Julian oscillation: Synthesis and summary. J. Geophys. Res. Atmos., 120, 4671-4689, https://doi.org/ 10.1002/2015JD023196.

Kuang, Z., 2008: A moisture-stratiform instability for convectively coupled waves. J. Atmos. Sci., 65, 834-854, https://doi.org/ 10.1175/2007JAS2444.1.

_ 2012: Weakly forced mock Walker cells. J. Atmos. Sci., 69, 2759-2786, https://doi.org/10.1175/JAS-D-11-0307.1.

Lau, W. K. M., and D. E. Waliser, Eds., 2012: Intraseasonal Variability in the Atmosphere-Ocean Climate System. 2nd ed. Springer-Verlag, $614 \mathrm{pp}$.

Liebmann, B., H. H. Hendon, and J. D. Glick, 1994: The relationship between tropical cyclones of the western Pacific and Indian Oceans and the Madden-Julian oscillation. J. Meteor. Soc. Japan, 72, 401-412, https://doi.org/10.2151/ jmsj1965.72.3_401. 
Madden, R. A., and P. R. Julian, 1971: Detection of a 4050 day oscillation in the zonal wind in the tropical Pacific. J. Atmos. Sci., 28, 702-708, https://doi.org/10.1175/15200469(1971)028<0702:DOADOI $>2.0$. CO; 2 .

$\longrightarrow$, and - 1972: Description of global-scale circulation cells in the tropics with a 40-50 day period. J. Atmos. Sci., 29, 1109-1123, https://doi.org/10.1175/1520-0469(1972)029<1109: DOGSCC $>2.0 . \mathrm{CO} ; 2$.

- - and ——, 1994: Observations of the 40-50-day tropical oscillation-A review. Mon. Wea. Rev., 122, 814-837, https://doi.org/10.1175/1520-0493(1994)122<0814:OOTDTO> 2.0.CO;2.

Majda, A. J., 2003: Introduction to PDEs and Waves for the Atmosphere and Ocean. Courant Lecture Notes in Mathematics, Vol. 9, American Mathematical Society, 234 pp.

—_, and M. G. Shefter, 2001: Models of stratiform instability and convectively coupled waves. J. Atmos. Sci., 58, 1567-1584, https://doi.org/10.1175/1520-0469(2001)058<1567:MFSIAC> 2.0.CO;2.

, and J. A. Biello, 2003: The nonlinear interaction of barotropic and equatorial baroclinic Rossby waves. J. Atmos. Sci., 60, 1809-1821, https://doi.org/10.1175/1520-0469(2003)060<1809: TNIOBA $>2.0 . \mathrm{CO} ; 2$.

- , and R. Klein, 2003: Systematic multiscale models for the tropics. J. Atmos. Sci., 60, 393-408, https://doi.org/10.1175/ 1520-0469(2003)060<0393:SMMFTT>2.0.CO;2.

—, and J. A. Biello, 2004: A multiscale model for the intraseasonal oscillation. Proc. Natl. Acad. Sci. USA, 101, 47364741, https://doi.org/10.1073/pnas.0401034101.

_ features of convective momentum transport. J. Atmos. Sci., 66, 373-392, https://doi.org/10.1175/2008JAS2805.1.

$\longrightarrow$, and $-2009 \mathrm{~b}$ : The skeleton of tropical intraseasonal oscillations. Proc. Natl. Acad. Sci. USA, 106, 8417-8422, https:// doi.org/10.1073/pnas.0903367106.

Maloney, E. D., 2009: The moist static energy budget of a composite tropical intraseasonal oscillation in a climate model. J. Climate, 22, 711-729, https://doi.org/10.1175/2008JCLI2542.1.

_- , and D. L. Hartmann, 2000: Modulation of hurricane activity in the Gulf of Mexico by the Madden-Julian oscillation. Science, 287, 2002-2004, https://doi.org/10.1126/ science.287.5460.2002.

Mapes, B. E., 1993: Gregarious tropical convection. J. Atmos. Sci., 50, 2026-2037, https://doi.org/10.1175/1520-0469(1993) $050<2026$ :GTC $>2.0$.CO;2.

- 2000: Convective inhibition, subgrid-scale triggering energy, and stratiform instability in a toy tropical wave model. J. Atmos. Sci., 57, 1515-1535, https://doi.org/10.1175/15200469(2000)057<1515:CISSTE > 2.0.CO;2.

Matsuno, T., 1966: Quasi-geostrophic motions in the equatorial area. J. Meteor. Soc. Japan, 44, 25-43, https://doi.org/10.2151/ jmsj1965.44.1_25.

Neelin, J. D., and I. M. Held, 1987: Modeling tropical convergence based on the moist static energy budget. Mon. Wea. Rev., 115, 3-12, https://doi.org/10.1175/1520-0493(1987)115<0003: MTCBOT $>2.0 . \mathrm{CO} ; 2$.

_ convective adjustment and the Madden-Julian oscillation. Part I: Analytical theory. J. Atmos. Sci., 51, 1876-1894, https://doi.org/10.1175/1520-0469(1994)051<1876:MOTVUC> 2.0.CO;2.

—_, and N. Zeng, 2000: A quasi-equilibrium tropical circulation model-Formulation. J. Atmos. Sci., 57, 1741-1766,
https://doi.org/10.1175/1520-0469(2000)057<1741:AQETCM> 2.0.CO;2.

_ I. M. Held, and K. H. Cook, 1987: Evaporation-wind feedback and low-frequency variability in the tropical atmosphere. J. Atmos. Sci., 44, 2341-2348, https://doi.org/10.1175/15200469(1987)044<2341:EWFALF>2.0.CO;2.

- O. Peters, and K. Hales, 2009: The transition to strong convection. J. Atmos. Sci., 66, 2367-2384, https://doi.org/10.1175/2009JAS2962.1.

Ogrosky, H. R., and S. N. Stechmann, 2016: Identifying convectively coupled equatorial waves using theoretical wave eigenvectors. Mon. Wea. Rev., 144, 2235-2264, https://doi.org/ 10.1175/MWR-D-15-0292.1.

$\longrightarrow, \ldots$, and S. Hottovy, 2019: Instability and nonlinear dynamics of the MJO in a tropical channel model with vertically varying convective adjustment. Theor. Comput. Fluid Dyn., 33, 307-323, https://doi.org/10.1007/s00162-019-00495-x.

Randall, D., 2012: Atmosphere, Clouds, and Climate. Princeton University Press, 277 pp.

Raymond, D. J., and Ž. Fuchs, 2009: Moisture modes and the Madden-Julian oscillation. J. Climate, 22, 3031-3046, https:// doi.org/10.1175/2008JCLI2739.1.

— , and M. J. Herman, 2011: Convective quasi-equilibrium reconsidered. J. Adv. Model. Earth Syst., 3, M08003, https:// doi.org/10.1029/2011MS000079.

— - S. L. Sessions, A. H. Sobel, and Z. Fuchs, 2009: The mechanics of gross moist stability. J. Adv. Model. Earth Syst., 1, 9, https://doi.org/10.3894/JAMES.2009.1.9.

Salby, M. L., R. R. Garcia, and H. H. Hendon, 1994: Planetaryscale circulations in the presence of climatological and waveinduced heating. J. Atmos. Sci., 51, 2344-2367, https://doi.org/ 10.1175/1520-0469(1994)051<2344:PSCITP > 2.0.CO;2.

Smith, L. M., and S. N. Stechmann, 2017: Precipitating quasigeostrophic equations and potential vorticity inversion with phase changes. J. Atmos. Sci., 74, 3285-3303, https://doi.org/10.1175/ JAS-D-17-0023.1.

Sobel, A., and E. Maloney, 2013: Moisture modes and the eastward propagation of the MJO. J. Atmos. Sci., 70, 187-192, https:// doi.org/10.1175/JAS-D-12-0189.1.

_ _ J. Nilsson, and L. M. Polvani, 2001: The weak temperature gradient approximation and balanced tropical moisture waves. J. Atmos. Sci., 58, 3650-3665, https://doi.org/10.1175/15200469(2001)058<3650:TWTGAA $>2.0$. CO;2.

_ - S. Wang, and D. Kim, 2014: Moist static energy budget of the MJO during DYNAMO. J. Atmos. Sci., 71, 4276-4291, https:// doi.org/10.1175/JAS-D-14-0052.1.

Stechmann, S. N., and A. J. Majda, 2009: Gravity waves in shear and implications for organized convection. J. Atmos. Sci., 66 , 2579-2599, https://doi.org/10.1175/2009JAS2976.1.

— dynamics. J. Atmos. Sci., 67, 3269-3285, https://doi.org/ 10.1175/2010JAS3380.1.

— , and H. R. Ogrosky, 2014: The Walker circulation, diabatic heating, and outgoing longwave radiation. Geophys. Res. Lett., 41, 9097-9105, https://doi.org/10.1002/2014GL062257.

_ , and A. J. Majda, 2015: Identifying the skeleton of the MaddenJulian oscillation in observational data. Mon. Wea. Rev., 143, 395-416, https://doi.org/10.1175/MWR-D-14-00169.1.

_- and S. Hottovy, 2017: Unified spectrum of tropical rainfall and waves in a simple stochastic model. Geophys. Res. Lett., 44, 10 713-10 724, https://doi.org/10.1002/2017GL075754.

_ A. J. Majda, and B. Khouider, 2008: Nonlinear dynamics of hydrostatic internal gravity waves. Theor. Comput. Fluid Dyn., 22, 407-432, https://doi.org/10.1007/s00162-008-0080-7. 
Takayabu, Y. N., 1994: Large-scale cloud disturbances associated with equatorial waves. I: Spectral features of the cloud disturbances. J. Meteor. Soc. Japan, 72, 433-449, https://doi.org/ 10.2151/jmsj1965.72.3_433.

Waliser, D. E., R. Murtugudde, P. Strutton, and J.-L. Li, 2005: Subseasonal organization of ocean chlorophyll: Prospects for prediction based on the Madden-Julian oscillation. Geophys. Res. Lett., 32, L23602, https://doi.org/10.1029/2005GL024300.

Wang, B., and H. Rui, 1990: Dynamics of the coupled moist Kelvin-Rossby wave on an equatorial beta-plane. J. Atmos. Sci., 47, 397-413, https://doi.org/10.1175/1520-0469(1990) 047<0397:DOTCMK > 2.0.CO;2.

, F. Liu, and G. Chen, 2016: A trio-interaction theory for Madden-Julian oscillation. Geosci. Lett., 3, 34, https://doi.org/ 10.1186/s40562-016-0066-Z.

Wheeler, M., and G. N. Kiladis, 1999: Convectively coupled equatorial waves: Analysis of clouds and temperature in the wavenumber-frequency domain. J. Atmos. Sci., 56, 374-399, https:// doi.org/10.1175/1520-0469(1999)056<0374:CCEWAO>2.0.CO;2.

Yang, D., 2018: Boundary layer diabatic processes, the virtual effect, and convective self-aggregation. J. Adv. Model. Earth Syst., 10, 2163-2176, https://doi.org/10.1029/2017MS001261. , and A. P. Ingersoll, 2013: Triggered convection, gravity waves, and the MJO: A shallow-water model. J. Atmos. Sci., 70, 2476-2486, https://doi.org/10.1175/JAS-D-12-0255.1.

, and - 2014: A theory of the MJO horizontal scale. Geophys. Res. Lett., 41, 1059-1064, https://doi.org/10.1002/ 2013GL058542.

Zhang, C., 2005: Madden-Julian Oscillation. Rev. Geophys., 43, RG2003, https://doi.org/10.1029/2004RG000158.

J. Gottschalck, E. D. Maloney, M. W. Moncrieff, F. Vitart, D. E. Waliser, B. Wang, and M. C. Wheeler, 2013: Cracking the MJO nut. Geophys. Res. Lett., 40, 1223-1230, https:// doi.org/10.1002/grl.50244. 